\title{
Acknowledgment to Reviewers of Biomolecules in 2020
}

\section{Biomolecules Editorial Office}

Citation: Biomolecules Editorial Office. Acknowledgment to Reviewers of Biomolecules in 2020. Biomolecules 2021, 11, 151. https:// doi.org/10.3390/biom11020151

Published: 25 January 2021

Publisher's Note: MDPI stays neutral with regard to jurisdictional claims in published maps and institutional affiliations.

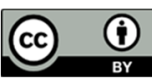

Copyright: (c) 2021 by the author. Licensee MDPI, Basel, Switzerland. This article is an open access article distributed under the terms and conditions of the Creative Commons Attribution (CC BY) license (http://creativecommons.org/licenses /by/4.0/).

MDPI AG, St. Alban-Anlage 66, 4052 Basel, Switzerland

Peer review is the driving force of journal development, and reviewers are gatekeepers who ensure that Biomolecules maintains its standards for the high quality of its published papers. Thanks to the cooperation of our reviewers, in 2020, the median time to first decision was 17 days and the median time to publication was 39 days. The editors would like to express their sincere gratitude to the following reviewers for their precious time and dedication, regardless of whether the papers were finally published:

Abate, Giulia

Abbott, Jessica K.

Abdelmohsen, Usama Ramadan

Abenavoli, Ludovico

Abeykoon, Jithma

Ablashi, Dharam

Abomoelak, Bassam

Abraham, Wolf-Rainer

Abramson, Hanley N.

Abushouk, Abdelrahman

Accardo, Angelo

Acquas, Elio

Adamczyk, Bartosz

Adamczyk-Grochala, Jagoda

Adamek, Jakub

Adamek, Mikolaj

Adams, James

Adams, Stephen

Adamska, Agnieszka

Adar, Sheera

Ådén, Jörgen

Adhikari, Neeta

Adriana, Estrada-Bernal

Affolter, Markus

Affourtit, Charles

Agamennone, Mariangela

Aggeli, Ioanna-Katerina

Aghaeepour, Nima

Agostino, Mark

Agüero-Chapin, Guillermin

Ahmad, Fahim

Ahn, Juhee

Ahn, Yeong Hee

Ai, Yong

Aiello, Francesca
Aimanianda, Vishukumar

Akai, Hiroyuki

Akbar, Mohammed

Akimov, Sergey A.

Akopova, Tatiana

Alam, Shafiul

Alarcón-Riquelme, Marta E.

Albani, Maria

Albarella, Sara

Albericio, Fernando

Albers, Sonja Verena

Alduina, Rosa

Alekseeva, Anna

Aleksova, Aneta

Alessenko, Alice V.

Alexandri, Maria

Alfano, Daniela

Al-Hilaly, Youssra

Ali, Hayssam M.

Alini, Mauro

Alipieva, Kalina

Allen, Claire

Allers, Thorsten

Allmang, Christine

Alloatti, Giuseppe

Almajano, María Pilar

Almeida-Paes, Rodrigo

Alonso, Alejandra D.

Alonso-Alconada, Daniel

Alseekh, Saleh

Altamirano-Bustamante, Myriam M.

Altegoer, Florian

Altieri, Fabio

Altintas, Mehmet M

Altman, Amnon 
Altman, Ryan A.

Alvarez Fernandez, María Antonia

Alves E Silva, Thiago Luiz

Alves, Ana Catarina

Alzubaidi, Laith

Amaral, Joana

Amaro, Helena

Amato, Dominick J.

Amato, Felice

Ambite, Ines

Ambrosino, Concetta

Ambrosio, Santiago

Ameln, Anne Klotzsche-von

Ames, James B

Amigo, Lourdes

Amir, Rachel

Amodio, Nicola

Amorós, Asunción

Amrani, Yassine

An, Hong

An, Jeung Hee

Anastasiadi, Maria

Anberitha Matthews, Anberitha

Anchordoquy, Tom

Ancuceanu, Robert

Anděra, Ladislav

Anders, Juanita

Andersen, Tonni Grube

Anderson, Marilyn A.

Andersson, Emma

André, Vânia

Andreas, Loren B

Andreev, Yaroslav A.

Andreoni, Francesca

Andres, Maria Fe

Andrés-León, Eduardo

Andrisano, Vincenza

Andrius, Kazlauskas

Angeletti, Mauro

Angioni, Alberto

Angius, Fabrizio

Anne, Jozef

Anni, Vedeler

Annibal, Andrea

Ansari, D. Mohd. Azam

Antalíková, Jana

Antinozzi, Cristina

Antipova, Veronica

Antoce, Arina Oana

Antognelli, Cinzia

Antoni, Gunnar
Antoniali, Giulia

Antoniassi, Rosemar

Antoniewska, Agata

Antony, Antoniou

Antonyuk, Svetlana

Appendino, Giovanni

Aprodu, Iuliana

Aprotosoaie, Ana Clara

Aquilano, Katia

Arai, Toshiro

Arakawa, Kenji

Araújo, Maria Eduarda Machado

Arbault, Stéphane

Archambault, Vincent

Arciello, Angela

Arcone, Rosaria

Ardalan, Maryam

Ardejani, Maziar

Arenas, Jesús

Arenas-Mena, Cesar

Arese, Marzia

Arguelles, Sandro

Arif, Tasleem

Arimura, Shinichi

Arizza, Vincenzo

Armanini, Decio

Arnaboldi, Paul

Arriagada, César

Arrizon, Javier

Arthur, Helen

Arvidsson, Per I.

Asa'ad, Farah

Asada, Hidetsugu

Asada, Ken

Asamizu, Shumpei

Aschtgen, Marie-stephanie

Asensio, Juan Luis

Ashaie, Maeirah

Ashihara, Eishi

Ashour, Mohamed Lotfy

Ashton, Nicholas W.

Assaf, Khaleel Ibrahim

Aszodi, Attila

Ataide, Sandro

Atanasova, Nina

Atreya, Chintamani D.

Attanzio, Alessandro

Attili, Anna Rita

Audrito, Valentina

Augustine, Josy

Auriemma, Giulia 
Autry, Joseph

Avdonin, Pavel V.

Aviles, Francesc Xavier

Aviñó, Anna

Ayabe, Tatsuhiro

Ayonrinde, Oyekoya T.

Ayyoob, Muhammad

Azarnia Tehran, Domenico

Azarov, Jan E.

Azevedo, Jorge

Azzini, Elena

Babichev, Sergii

Babu, Gopal J.

Bąchor, Remigiusz

Bacik, John-Paul

Back, Kyoungwhan

Badea, Mihaela

Bade-Doeding, Christina

Badu-Tawiah, Abraham

Baggiani, Claudio

Baglietto-Vargas, David

Bagnard, Dominique

Bagoly, Zsuzsa

Bahuguna, Ashutosh

Bai, Shuhua

Baiesi, Marco

Bailey, Charles G

Bailly, Benjamin

Bajoghli, Baubak

Bajusz, Dávid

Bakaeva, Zanda

Bakhti, Mostafa

Bakillah, Ahmed

Balan, Prabhu

Balcerczak, Ewa

Balcerek, Maria

Baldo, Guilherme

Balestra, Costantino

Ball, Zachary T.

Baltina, Lidia

Balzano, Tiziano

Ban, Bhupal

Banerjee, Santanu

Banfalvi, Zsofia

Bang, John J.

Baniene, Rasa

Banito, Ana

Bañuls, Maria Jose

Bao, Jinku

Bao, Jun-Xiang

Baptista, Mafalda S.
Barabutis, Nektarios

Baraldi, Elena

Baranowska-Kuczko, Marta

Barault, Ludovic

Barbagallo, Davide

Barbosa, Armenio

Barchetta, Ilaria

Barderas, Rodrigo

Bärebring, Linnea

Barfield, Robyn

Barker, Tyler

Barnea, Eytan R

Barnett, Susan

Bar-Or, David

Barranco, Isabel

Barreto, Guillermo

Barrett, James

Barros, Marcelo Paes

Barshtein, Gregory

Barta, Csengele

Bartolazzi, Armando

Bartolini, Manuela

Bartolini, Paolo

Bartolotti, Massimo

Barton, Matthew J

Bartova, Eva

Bartuzi, Damian

Barvik, Ivan

Barygin, Oleg I.

Basak, Debasish

Basilio Heredia, Jose

Basini, Giuseppina

Basith, Shaherin

Bassanini, Ivan

Basso, Daniela

Bassolino, Laura

Bateman, Andrew

Batissoco, Ana C.

Batsché, Eric

Battistelli, Michela

Baucum, Anthony J.

Baudier, Jacques

Bauer, Johann

Bauer, Stefanie

Baumgartner, Ulrich

Bautista, Marta

Bax, Bridget E.

Bayascas, Jose R

Bayer, Peter

Bayfield, Mark

Beale, David 
Beamer, Lesa J.

Becciolini, Andrea

Becker, Thomas

Beckett, Dorothy

Bednarek, Piotr

Bednárová, Lucie

Beerhues, Ludger

Begemann, Gerrit

Beharry, Kay D.

Beilby, Mary

Bekatorou, Argyro

Belayew, Alexandra

Bełdowski, Piotr

Belfiore, Antonino

Belin De Chantemèle, Eric J.

Bellier, Jean Pierre

Belluzzi, Elisa

Beltowski, Jerzy

Belyi, Yury

Benabdellah, Karim

Benarba, Bachir

Benard, Giovanni

Bendas, Gerd

Benedec, Daniela

Benevolenskaya, Elizaveta

Benfenati, Valentina

Benistant, Christine

Bennett, James P.

Bennett, Robert G.

Benohoud, Meryem

Berdis, Anthony J.

Berenguer, Jose

Berenson, James

Berenyiova, Andrea

Berger, Trish

Bergler, Helmut

Berliner, Lawrence J.

Bernabéu, Carmelo

Bernal, Freddy Alexander

Bernardes-Génisson, Vania

Bernardi, Tatiana

Bernstein, Nirit

Berthold-Pluta, Anna

Berthoud, Viviana M

Berti, Federico

Bertin, Matthew J.

Bertolotti, Marco

Bertrand, Czarny

Besenfelder, Urban $\mathrm{H}$.

Bessho, Tadayoshi

Beuning, Penny
Beverly, Levi J.

Bevilacqua, Vitoantonio

Beyer, Eric $C$.

Bhandari, Manohar Prasad

Bharadwaj, Shiv

Bhat, Kamakoti

Bhattacharya, Abhisek

Bhattacharya, Supriyo

Bhoobalan-Chitty, Yuvaraj

Biagi, Marco

Bianchi, Vittorio

Bielczyk-Maczyńska, Ewa

Biener, Moritz

Bignon, Christophe

Bijak, Michał

Bikiaris, Dimitrios

Bilal, Muhammad

Bileck, Andrea

Bills, Gerald F.

Bilska-Wilkosz, Anna

Bilski, Jan

Biondi, Barbara

Birková, Anna

Bischof, Joachim

Bischof, Johannes

Bishop, Roger

Bisig, Christoph

Bisson, Alexandre

Bitto, Alessandra

Bizon, Katarzyna

Black, Esther P.

Blakely, William F.

Blanco Fernandez, Matias

Blanco, Eduardo

Blanco, Pilar

Blanquart, Christophe

Blasiak, Janusz

Blumenschein, Tharin

Boccaccini, Alessandra

Boccuni, Fabio

Bochenkova, Anastasia V.

Bocian, Szymon

Bock, Florian

Boer, Vincent C. J. De

Boffano, Paolo

Bogdanov, Alexey A.

Böhmer, Frank

Bohn, Erwin

Bojarová, Pavla

Bojarska, Joanna

Bojarski, Andrzej 
Boland, Sebastian

Boldingh, Helen

Bolotin, Monique

Bolt, Edward L.

Bölükbas, Deniz

Bomken, Simon

Boncela, Joanna

Bonciu, Elena

Bonet, Esther Udina

Bonfiglio, Rita

Bonfio, Claudia

Bonifacio, Maria Addolorata

Bonomini, Mario

Bontems, François

Bonuccelli, Gloria

Booth, Stephanie

Borges, Ricardo

Borgnia, Mario J.

Boris, Pejin

Boroń, Dariusz

Boronat, Anna

Borota, Ana

Borovac, Josip A.

Borse, Vikrant

Borst, Jan Willem

Borysiak, Slawomir

Bosak, Anita

Bose, Debojit

Bossche, Jan Van Den

Bossis, Guillaume

Bosso, Andrea

Bottani, Emanuela

Bottini, Massimo

Bougard, Daisy

Bouitbir, Jamal

Bounaceur, Roda

Bourdon, Emmanuel

Bourgault, Steve

Bourgougnon, Nathalie

Bouschet, Tristan

Boutelle, Martyn G.

Bovin, Nicolai

Bovolin, Patrizia

Bowen, Mark E.

Bowman, Daryl

Boysen, Reinhard

Bozzaro, Salvatore

Bracco, Enrico

Bradbury, Allison

Brahmachari, Saurav

Braicu, Cornelia
Brand, Izabella

Brandenburg, Lars Ove

Brandl, Christopher J.

Brannick, Erin M.

Brasó-Maristany, Fara

Braun, Ralf

Bravo-Sanchez, M. G.

Breckpot, Karine

Bregier-Jarzębowska, Romualda

Brem, Jurgen

Bren, Urban

Brendel, Johannes

Brennan, Caroline

Brenowitz, Michael

Bresolí-Obach, Roger

Brestic, Marian

Breuzard, Gilles

Briedé, Jacco

Briers, Yves

Brighenti, Virginia

Brígido, Clarisse

Brindisi, Matteo

Briza, Peter

Broda, Małgorzata A.

Brodolin, Konstantin L.

Brogi, Simone

Bromage, Erin

Bronstein, Jeff

Brosius, Jürgen

Brown, Ashley N.

Brown, Lindsay

Brown, Paul B

Brown, Robert J.

Brozmanová, Mariana

Brozyna, Anna

Bru, Samuel

Bruchmann, Sebastian

Brückner, Adrian

Bruins, Jantinus H.

Brull, Astrid

Brunetti, Angela

Brunetti, Luigi

Brunetti, Oronzo

Bruno, Stefania

Bruzzone, Santina

brzoska, malgorzata michalina

Bubici, Giovanni

Buchberger, Alexander

Budzisz, Elżbieta

Bueno-Silva, Bruno

Bukhari, Marwan A.S. 
Bukrinsky, Michael

Bulati, Matteo

Bulc, Michał

Bunea, Andrea

Bungau, Simona

Bungau, Simona Gabriela

Bungert, Jörg

Bunik, Victoria

Buonaguro, Elisabetta Filomena

Burbage, Marianne

Burch-Smith, Tessa

Burcul, Franko

Burger, Michael C

Burgos, Héctor

Burguete, Maria Consuelo

Burke, Andrew Robert

Burkhead, Jason

Bürklein, Sebastian

Burlacu, Alexandru

Burpo, Fred J.

Burton, Zachary F.

Burvenich, Ingrid

Busath, David D.

Busch, Albert

Bussmann, Rainer W.

Bustaffa, Elisa

Bustos, Diego Martin

Butcher, Adrian

Buzoianu, Anca

Bydlowski, Sérgio Paulo

Byeon, Haewon

Byrne, Bernadette

Byrne, Mark E.

Bystrom, Jonas

Bystrowska, Beata

Byun, Sanguine

Cabaro, Serena

Cacanyiova, Sona

Caceres, Javier

Cádiz-Gurrea, María De La Luz

Cafiero, Mauricio

Cagnin, Stefano

Cai, Kai

Cairrão, Elisa

CAKIR, Birsen

Cal, Santiago

Calarco, Anna

Caldara, Marina

Caldwell, Gary

Callaghan, David

Caltabiano, Rosario
Calvano, Cosima D.

Calvo Guirado, José Luis

Cameron, Angus

Cameron, Donald

Cameron, Simon

Cametti, Cesare

Camila, Hochman-Mendez

Camire, Mary

Campbell, Grace

Campilho, Ana

Campomenosi, Paola

Camporesi, Enrico M.

Campos Rosa, Joaquín María

Canals, Isaac

Canchaya, Carlos

Candéias, Serge

Candela, Diaz-Canestro

Canela, Enric I.

Cannas, Angela

Canonico, Barbara

Canonico, Laura

Cao, Jian

Cao, Wei

Cao, Xian

Capasso, Raffaele

Capece, Angela

Caplan, Allan

Capozzi, Vittorio

Cappello, Paola

Caprio, Massimiliano

Capuano, Vittorio

Caputa, Michał

Caputo, Lucia

Carazo, Alejandro

Carboni, Lucia

Cardellicchio, Cosimo

Cardenas, Marité

Cardona, Fernando

Cardoso, Susana M.

Caretta, Antonio

Cariani, Elisabetta

Carlisi, Daniela

Carlone, Robert L.

Carlos, Sao-Jose

Carman, George M.

Carmichael, Gordon G

Carmona-Saez, Pedro

Carnevali, Davide

Carone, Benjamin R.

Carradori, Simone

Carrieri, Antonio 
Carrizzo, Albino

Carro, Eva

Carter, Wayne

Carugo, Oliviero

Carullo, Gabriele

Caruntu, Constantin

Caruso, Francesco

Carvalho Pimenta, Daniel

Carvalho, António Paulo

Carver, John A.

Casampai, Antal

Casapullo, Agostino

Casar, Berta

Casas, Rosa M.

Casella, Luigi

Casillas, Sonia

Caspari, Thomas

Cassinelli, Giuliana

Castaing, Bertrand

Castiglia, Daniela

Castilla, Joaquin

Castillo, Antonio

Castillo, Francisco

Castillo-Tong, Dan Cacsire

Caston, José $\mathrm{R}$

Castresana, Javier S.

Castro, Mariana S.

CASTRONUOVO, Donato

Caswell, Clayton C.

Catalan, Marcelo

Catalani, Elisabetta

Catanzaro, Elena

Catanzaro, Giuseppina

Cathy, Jackson

Caughey, Byron

Cavaliere, Gina

Cavallo, Gisella

Cavazza, Christine

Caviglia, Gian Paolo

Cavoretto, Paolo

Caza, Tiffany

Ceci, Luigi R.

Celebioglu, Asli

Cembrowska-Lech, Danuta

Cendron, Laura

Cerdeira, Cláudio Daniel

Cereda, Matteo

Cerezo, Javier

Černá, Marie

Černý, Jiří

Ceron-Carrasco, Jose
Cerrito, Maria Grazia

Cervellati, Franco

Cervera, Ana

Cervetto, Chiara

Céspedes, María

Cevenini, Armando

Chachami, Georgia

Chadwick, Ruth

Chagas, Juana C. Cariri

Chaimbault, Patrick

Chakrabarti, Sutapa

Chambers, Jeremy W.

Chan, Yau Kei

Chan, Yin-Ching

Chand, Kirat K.

Chandrasekaran, Arun Richard

Chang, Chia-che

Chang, Fei

Chang, Kee-lung

Chang, Ling-Chu

Chang, Long-Sen

Chang, Margaret Dah-tsyr

Chang, Te-Sheng

Chang, Wen-Chi

Chang, Yuan-Yen

Chang, Yu-Wei

Chao, Yun-Yang

Chapoval, Svetlana P.

Charbonneau, Michel

Charreau, Beatrice

Chatterjee, Madhumita

Chaudhari, Kiran

Chaudhry, Bill

Chaudhry, Rasul

Chauhan, Neeraj

Chaumont Dubel, Severine

Chaurasia, A. K.

Chauvot De Beauchene, Isaure

Chavez-Santoscoy, Rocio Alejandra

Chazin, Walter

Checconi, Paola

Checcucci, Alice

Cheemarla, Nagarjuna Reddy

Cheignon, Clémence

Cheloha, Ross

Chen, Chin-Chu

Chen, Chung-Ming

Chen, Cortina

Chen, $\mathrm{Hu}$

Chen, Jen-Tsung

Chen, Ji-Yih 
Chen, Lih-Geeng

Chen, Muyuan

Chen, Pan

Chen, Po-Yuan

Chen, Qi

Chen, Qiusheng

Chen, Qun

Chen, Ray-Bing

Chen, Rong-Jane

Chen, Shuai

Chen, Vincent

Chen, Xingqi

Chen, Xixian

Chen, Ya

Chen, Yann-Jang

Chen, Yi-Hau

Chen, Ying

Chen, $\mathrm{Yu}$

Chen, Zhao

Cheng, Chia-Hsiung

Cheng, Chi-Lien

Cheng, Ching-Yi

Cheng, Haili

Cheng, Hong Sheng

Cheng, Juei-tang

Cheng, Wei-chieh

Cheng, Xiaoming

Cheng, Yuan-Bin

Chengwei, Yang

Chen-Hui, Chen

Chérel, Isabelle

Chern, Edward

Chernikov, Oleg

Chernova, Tatiana

Cheung, David L.

Cheungpasitporn, Wisit

Chew, Nick Guan Pin

Chi, Ya-Hui

Chiang, Hung-Lung

Chiappini, Franck

Chiariotti, Lorenzo

Chibbar, Ravindra

Chien, Chih-Yen

Chien, Peter

Chieppa, Marcello

Chin, Kok Yong

Chin, Young-Won

Chini, Andrea

Chino, Marco

Chirikova, Nadezhda K.

Chirumbolo, Salvatore
Chittori, Sagar

Chiummiento, Lucia

Chlubek, Dariusz

Chmurzyńska, Agata

Cho, Hyosun

Cho, Jung Sook

Cho, Namki

Cho, Yongyeon

Choi, Hyung-Kyoon

Choi, Sangchun

Choi, Yoon Kyung

Choromanska, Anna

Chou, Yachang

Choudhury, Swarup Roy

Chow, Christine S.

Chow, Kwok-Fan

Chowdhury, Ezharul Hoque

Chowdhury, Farhan

Christodoulou, Maria-Ioanna

Christoforides, Elias

Chuang, Hung-Yi

Chubiz, Lon

Chueh, Pin Ju

CHUI, Kwok Tai

Chun, Sungkun

Chung, Sungjin

Chung, Won-Yoon

Cianciosi, Danila

Ciandrini, Luca

Ciani, Maurizio

Ciaudo, Constance

Cicala, Carla

Cichero, Elena

Cieplak, Marek

Ciesielski, Slawomir

Cifuentes, Daniel

Cilla, Antonio

Cimadevilla, Jose

Cinar, Bekir

Cindrić, Mario

Cioanca, Oana

Ciornea, Elena Todirascu

Cipolletti, Manuela

Ciregia, Federica

Cirrincione, Girolamo

Citti, Cinzia

Clara, Mattu

Clarke, Anthony J.

Clarke, Chirstopher

Clarke, Jonathan

Clarke, Stephen 
Claus, Harald

Clemens, Roger

Clementi, Catia

Clichici, Simona

Cloninger, Mary

Close, Pierre

Cocco, Tiziana

Cockburn, Darrell

Coelho-Silva, Juan

Coenen, Marieke J.H.

Coetzee, William A.

Coffman, Lan

Cohen, Seth

Coin, Irene

Coisson, Jean Daniel

Coleine, Claudia

Colige, Alain

Collavin, Licio

Colliec-Jouault, Sylvia

Collins, John D.

Colomba, Paolo

Colomina, Maria Teresa

Colone, Marisa

Colpitts, Che C.

Colston, Timothy J.

Coluccia, Antonio

Colucci-D'Amato, Luca

Colussi, Gian Luca

Colussi, Silvia

Comai, Stefano

Comoy, Emmanuel

Conflitti, Paolo

Conrad, David M

Constantinescu-Aruxandei, Diana

Consumi, Marco

Conte, Maria Sasi

Conti, Bice

Contreras-Cornejo, Hexon Angel

Convertini, Paolo

Conway, Edward

Cooke, Amy

Copic, Alenka

Copolovici, Dana Maria

Copolovici, Lucian

Corazzari, Marco

Corbeil, Denis

Cordeiro, Yraima

Cordero, Chiara Emilia

Cordonnier, Agnès

Cornejo, Alfonso

Cornelison, Christopher T.
Corr, Sinéad

Corsaro, Alessandro

Corzo-León, Dora E.

Cosentino, Nicola M.

Costa De Camargo, Adriano

Costa, Joana

Costa, Marina C.

Costa, Raquel

Costa, Roberto

Cote, Christopher

Cotignola, Javier

Couillaud, Franck

Courtois, Gilles

Coyle, Patricia K.

Cozza, Giorgio

Crehuet, Ramon

Crépeaux, Guillemette

Crespo-Hernández, Carlos

Crisafulli, Giovanni

Crisponi, Guido

Cristofori-Armstrong, Ben

Crosas, Bernat

Crosson, Sean

Crowder, Michael

Cruz, Carla

Cruz, Rebeca

Csapo, Edit

Cséplő, Ágnes

Csupor, Dezső

Cubellis, Maria

Cubero, Francisco Javier

Cuff, Simone

Cui, Haissi

Cui, Huaqing

Cui, Hui

Cui, Qingbin

Cui, Xiaoying

Cummings, Brian

Cunha, Rodrigo A.

Cunningham, Christopher W.

Cuppoletti, John

Curi-Borda, Cecilia K.

Curnow, Paul

Curtin, Nicola J

Cutignano, Adele

Cuya, Ricardo Teobaldo

Cuzzolin, Alberto

Czarnocki, Zbigniew

Czechowska, Joanna

Czernel, Grzegorz

Czogalla, Aleksander 
Czumaj, Aleksandra

Czyż, Katarzyna

Czyżnikowska, Żaneta

D. Bortner, Carl

D'Amelio, Raffaele

D'Anneo, Antonella

D'Arcy, Padraig

D’arcy, Pádraig

D'Elia, Domenica

D'Errico, Stefano

D'Onofrio, Nunzia

D'Orazi, Gabriella

Da Silva, Gabriela Jorge

Daberdaku, Sebastian

Daci, Armond

Dadakova, Eva

Daehn, Ilse

Dai, Yumin

Dal Prá, Ilaria

Dalby, Kevin N.

Dall'Acqua, Stefano

Damiano, Fabrizio

Danac, Ramona

Danesi, Francesca

Dangles, Olivier

Daniellou, Richard

Danilo, Cucchi

Daragó, Adam

Darenskaya, M. A.

Darling, Andrea L.

Daroch, Maurycy

Das, Anindita

Das, Joydip

Das, Viswanath

Dash, Biraja

Dashti, Yousef

Daughdrill, Gary W.

Davies, Christopher

Davies, Lindsay

Davignon, Jean-luc

Davis, Fred

Davuluri, Gangarao

Dawood, Mahmoud A.O.

Day, Catherine L.

De Angelis, Anna

De Araujo, Elvin

De Bellis, Luigi

De Craene, Johan-Owen

De Crécy-Lagard, Valérie

De Deurwaerdere, Philippe

De Falco, Enrica
De Feo, Vincenzo

De Giorgi, Maria Luisa

De Guia, Roldan M.

De La Luz Garcia-Hernandez, Maria

De La Vega, Laureano

De Lago, Eva

De Ligt, Joep

De Los Rios, Cristobal

De Luca, Anastasia

De Marco, Ario

De Masi, Luigi

De Molina, Ana Ramírez

De Moreno, A

De Paepe, Boel

De Pinto, Vito

De Rasmo, Domenico

De Rosa, Salvatore

De Vega, Antonio

De Vita, Daniela

DeAngelis, Paul

Del Re, Marzia

Delbarre Ladrat, Christine

Dell'Orco, Daniele

Dell'Oste, Valentina

Della Sala, Gerardo

Delogu, Giovanna

Dempfle, Astrid

Dempsey, Christopher E.

Deng, Hai

Deng, Qiliang

DeNicola, Gina

Dent, Joseph A.

Denvir, Martin A.

Dergunov, Sergey

Deseure, Kristof

Deshmukh, Rupesh

Deshpande, Gauravi

Deslouches, Berthony

Desmet, Tom

Deth, Richard

Detmar, Michael

Dever, Jane

Deyev, Igor E.

Dhaenens, Claire-Marie

Dhankher, Om Parkash

Dhar, Debanjan

Dharmaraj, Selvakumar

Di Bussolo, Valeria

Di Dato, Valeria

Di Filippo, Marzia

Di Fiore, Anna 
Di Foggia, Michele

Di Fonzo, Alessio

Di Giuda, Daniela

Di Liegro, Italia

Di Maro, Antimo

Di Martino, Maria Teresa

Di Martino, Orsola

Di Micco, Simone

Di Michele, Alessandro

Di Rocco, Giuliana

Di Sotto, Antonella

Diaferia, Carlo

Dias, Juliana

Diaz, Arturo

Díaz, Caridad

Didangelos, Triantafyllos

Diepold, Andreas

Dietz, Allan B.

Dijana Škorić, Dijana

Dijkstra, Bauke W.

Dikeakos, Jimmy

Dimasi, Nazzareno

Diminic, Janko

Dimitroff, Charles J.

Ding, Yousong

Diomede, Francesca

Diomede, Luisa

Dissel, Stephane

Dittadi, Ruggero

Dittmar, Gunnar

Dittmer, Jurgen

Divella, Rosa

Djabali, Karima

Djamgoz, Moustafa

Djedaïni-Pilard, Florence

Djokovic, Radojica

Długaszewska, Jolanta

Długosz, Ewa

Dmitryjuk, Małgorzata

Dmitrzak-Węglarz, Monika

Do, Thuy

Dobrikova, Anelia G.

Dobryszycki, Piotr

Docea, Anca Oana

Docimo, Teresa

Doda, Sai Reddy

Dolashka, Pavlina

Doležal, Karel

Doligalska, Maria

Dolzhenko, Anton V.

Domingo-Almenara, Xavier
Domingos, Pedro M.

Domingues, Pedro

Domingues, Sara

Domitrović, Robert

Donati, Ivan

Donato, Rosario

Dong, Shen

Dong, Yufeng

Donno, Dario

Dontsova, Olga A.

Dormoy, Valerian

Dorotkiewicz-Jach, Agata

Dos Santos, Catarina

Dos Santos, Patricia

Dos Santos, Reinaldo Sousa

Dosio, Franco

Dosztanyi, Zsuzsanna

Douki, Thierry

Douma, Mountasser

Doyen, Alain

Doytchinova, Irini

Dräger, Gerald

Dragoš, Anna

Dreschers, Stephan

Dreyfus, Georges

Drin, Guillaume

Drobizhev, Mikhail

Drolet, Barbara S.

Drori, Ran

Drosten, Matthias

Drummond, Heather

Druzbicki, Kacper

Drużyńska, Beata

Du Plessis, Heinrich W

Duarte, Noelia

Dubiel, Wolfgang

Dubrez, Laurence

Ducaiova, Zuzana Kovalikova

Duchateau, Nicolas

Dudová, Iva

Duenas, Montserrat

Duennwald, Martin L

Dufour, Antoine

Dullaart, Robin Pieter Frank

Dumas, Philippe

Dumetz, Franck

Dunn, Michael F.

Dupont, Joëlle

Durek, Thomas

Dursun, Serdar

Dutta, Arijit 
Dutta, Palash

Dutta, Rahul

Dzhemileva, Lilya

Dziendzikowska, Katarzyna

E Quelle, Dawn

Eamens, Andrew L.

Ebani, Valentina Virginia

Ebel, Frank

Ebihara, Lisa

Ebrahim, Weaam

Echeverria, Fernando

Eckhart, Leopold

Ediriweera, Meran Keshawa

Eduati, Federica

Edwards, Dylan R.

Efimova, Evgeniya V.

Egea, Javier

Eggeling, Lothar

Egger, Boris

Ehata, Shogo

Eide, David

Eiring, Anna

Eisel, Ulrich L. M.

Eisenbach, Michael

Ejidike, Ikechukwu P.

Ekielski, Adam

EkVitorin, Jose

El-Amri, Chahrazade

Elbayoumi, Tamer A

Elble, Randolph C.

Eldeeb, Mohamed

Eleftheriadis, Theodoros

Eleftheriou, Cyril

Elfalleh, Walid

Elizondo-Montemayor, Leticia

Ellgaard, Lars

Ellis, Lee D.

El-Maarouf-Bouteau, Hayat

ElMallah, Mai K.

Elosegui-Artola, Alberto

Emilsson, Valur

Emmert, Steffen

Endres, Kristina

Engelberg, David

Engelberth, Jurgen

Engelhardt, Paul Friedrich

Engler, Anna

Enos, Reilly T.

Enyedi, Kata Nóra

Erdmann, Kai Sven

Eriani, Gilbert
Erickson, Peter

Erikson, Keith

Erjavec, Gordana Nedic

Eroglu, Abdulkerim

Ersilia, Alexa

Erythropel, Hanno C.

Esatbeyolgu, Tuba

Espino, Javier

Esposito, Francesco

Esquifino, Ana Isabel

Esseltine, Jessica

Esser, Jennifer

Estevez, Alicia

Estévez, Jorge

Ethirajan, Anitha

Eulenburg, Volker

Evgen'ev, Mikhail B

Evidente, Antonio

Eyer, Luděk

Ezhov, Marat

Ezquerra-Brauer, Josafat-Marina

Fabiani, Roberto

Fabris, Linda

Facelli, Julio C

Factor-Litvak, Pam

Faenza, Irene

Faísca, Patrícia

Falanga, Annarita

Falciani, Chiara

Fällman, Maria

Fallowfield, Jonathan

Fang, Cheng

Fang, Evandro F.

Fang, Qingming

Fang, Xianjun

Fang, Xing

Fania, Luca

Fanizzi, Francesco Paolo

Fantini, Elio

Farmaki, Theodora

Farmer, Diana

Farris, Alton Brad

Farsky, Sandra H.p.

Fato, Romana

Fauconnier, Marie-Laure

Faudry, Eric

Favre-Réguillon, Alain

Fazakerley, Daniel

Fedorova, Olga V.

Fei, Tong

Feintuch, Akiva 
Feith, David

Feldman, Ricardo

Feldo, Marcin

Feliciello, Isidoro

FÉLIX, RUTE CASTELO

Feng, Hua-Jun

Fentiman, Ian

Ferguson, Matthew L.

Fernandes, João Paulo S.

Fernandes, Rosa Cristina Simoes

Fernandes, Tiago G.

Fernández Ochoa, Álvaro

Fernández, María Dolores

Fernández-Álvarez, Alfonso

Fernandez-Jimenez, Nora

Fernández-Moreno, Mercedes

Fernandez-Palomo, Cristian

Fernandez-Recio, Juan

Fernández-Rodríguez, Javier

Fernandez-Vizarra, Erika

Fernig, David

Ferraguti, Francesco

Ferrao, Petranel

Ferrari, Marco

Ferraroni, Marta

Ferreira, Christina

Ferreira, Helena

Ferreira, Leonardo G.

Ferreira, Marcelo

Ferreras, José M.

Ferreri, Carla

Ferrer-Montiel, Antonio

Ferri, Jose M.

Ferri, Maura

Ferro, Emer Suavinho

Fich, Eric A.

Fiester, Steve

Figueroa-Espinoza, Maria Cruz

Filek, Maria

Filgueiras, Camila

Filip, Adriana

Filipeanu, Catalin

Filipovic, Miodrag

Fillmore, Helen

Finck, Brian N.

Finol-Urdaneta, Rocio K.

Fiore, Vincenzo

Fiori, Mariana

Fisar, Zdenek

Fischer, Henrike

Fischer, Reinhard
Fischer, Utz

Fisher, Jed

Fisher, Paul

Fisk, John D.

Fiszer, Agnieszka

Fitter, Jörg

Fitzgerald, David

Fizeșan, Ionel

Florán, Benjamín

Florek, Ewa

Flores, Ignacio

Flórez-Fernández, Noelia

Floris, Matteo

Florsheim, Esther

Fondufe-Mittendorf, Yvonne N.

Fonin, Alexander

FONTANA, GIANFRANCO

Fontés, Michel

Ford, Lauren

Forest, Fabien

Forini, Francesca S.

Forlani, Fabio

Formenti, Federico

Forte, Elena

Forte, Maurizio

Fossa, Paola

Fradin, Chantal

Fragoso, Alex

Fraietta, Renato

Franca, Eduardo F

Franca, Tanos Celmar Costa

Francelle, Laetitia

Franceschini, Nicola

Francisco, Tânia

Francklyn, Christopher S.

Francuzik, Wojciech

Frank, Craig L.

Frank, Saša

Franke, Jakob

Franzoni, Giulia

Fratantonio, Deborah

Frau, Juan

Frecer, Vladimir

Freeman, Michael F.

Fretté, Xavier

Frick, Luciana

Fricker, Rosemary

Friedman, Thomas

Fröhlich, Eleonore

Frugis, Giovanna

Fu, Beide 
Fu, Peng

$\mathrm{Fu}$, Shu-Ling

$\mathrm{Fu}$, Zheng

Fujioka-Kobayashi, Masako

Fujita, Masaki

Fujita, Mikako

Fujita, Wakako

Fukuchi, Satoshi

Fukuda, Kenji

Fukuda, Seiji

Fukudome, Akihito

Fuliaş, Adriana Violeta

Fumiaki, Ito

Furneri, Pio Maria

Furse, Samuel

Furukawa, Koichi

Gabbia, Daniela

Gábor, Paragi

Gadermaier, Gabriele

Gaebel, Ralf

Gaedcke, Jochen

Gaetani, Lorenzo

Gagliano, Nicoletta

Gagliano, Teresa

Gaidano, Valentina

Gaj, Renata

Gál, Peter

Gala, Rikhav P

Galardi, Silvia

Galdiero, Maria Rosaria

Galeazzi, Roberta

Galindo, Máximo Ibo

Galkina, Svetlana I.

Gall Troselj, Koraljka

Gallagher, Emily Jane

Gallant, Nathan

Gallegos Monterrosa, Ramses

Gallegos-Arreola, Martha Patricia

Gallo, Gianluca

Gallo, Monica

Galluccio, Michele

Galpert, Deborah

Galvan, Daniel L

Gama, Sofia

Gan, Samuel

Gan, Wenjian

Gangadaran, Prakash

Gao, Bei

Gao, Ju

Gao, Zhe

García Fernández, Emilio
García Gonzalo, Francesc

García Mateu, Mauricio

Garcia, Samuel

García-Angulo, Penélope

Garcia-Bailo, Bibiana

Garcia-Borron, Jose Carlos

Garcia-Bustos, Jose F.

García-Cañadilla, Patricia

Garcia-Gallego, Sandra

García-García, Rosa María

García-González, Víctor

Garcia-Jares, Carmen

García-Malinis, Ana Julia

García-Ortega, Lucía

Garcia-Pardo, Javier

Garcia-Vallve, Santiago

Garner, Bianca L.

Garozzo, Domenico

Garrett Da Costa, Rafael

Garrido, Saulo

Garriga, Pere

Gasic, Ivana

Gašić, Uroš M.

Gáspári, Zoltán

Gaszner, Balázs

Gatto, Francesca

Gauld, James W.

Gaustad, Ann Helen

Gautieri, Alfonso

Gavara, Núria

Gavín, Rosalina

Gavira, José

Gaweł-Bęben, Katarzyna

Gawlik-Dziki, Urszula

Gawri, Rahul

Ge, Wanzhong

$\mathrm{Ge}$, Xin

Geisler-Lee, Jane

Gell, David A.

Geller, Herbert M.

Gelpi, Josep Lluis

Gemma, Sandra

Gendaszewska-Darmach, Edyta

Genetos, Damian C

Genilloud, Olga

Genisheva, Zlatina

Georgiades, Savvas

Georgiev, Vasil

Gerard, Helene

Gerdol, Marco

Gergs, Ulrich 
Germain, Pierre

German, Nadezhda A.

Gessner, Guido

Geszke-Moritz, Małgorzata

Ghavami, Saeid

Ghayor, Chafik

Ghigo, Alessandra

Ghirga, Francesca

Ghodake, Gajanan

Ghosh, Sanchita

Ghysels, An

Giacomazza, Daniela

Giamperi, Laura

Giampietro, Letizia

Giannoni, Patrizia

Giannotti, Marina Inés

Giansanti, Francesco

Gibbs, Bernhard

Gil, Jesus Perez

Giles, Gregory

Giles, Wayne

Giliopoulos, Dimitrios

Gillespie, James W.

Gillet, Germain

Gillingham, Melanie B.

Gilmour, Susan

Gimpel, Matthias

Ginsberg, Stephen D.

Giordano, Antonio

Giordano, Samantha

Giorgio, Selma

Giovagnoli, Raffaela

Giovannelli, Pia

Giovannoni, Roberto

Giovarelli, Mirella

Giraffa, Giorgio

Giraldo, Jesús

Girao, Henrique

Giri, Hemant

Gitlin-Domagalska, Agata

Giudetti, Anna

Giuffrida, Paolo

Giuliano, Michela

Gjörloff Wingren, Anette

Gladysheva, Inna P.

Glass, Karen C.

Glatt, Sebastian

Glazer, Lilah

Gloess, Alexia N.

Glover, Kerney Jebrell

Glynn, Steven
Gnoni, Antonio

Godlewski, Grzegorz

Godzien, Joanna

Goff, Wilfried Le

Gohda, Eiichi

Goldberg, Alfred

Goldeman, Waldemar

Goldsmith, Elizabeth

Golemi-Kotra, Dasantila

Golenberg, Edward M.

Golombick, Terry

Gomer, Richard

Gomes, Andreia

Gomes, Bruno Costa

Gomes, Joao R

Gomes, Nelson

Gomes, Pedro

Gómez De Cedrón, Marta

Gomez-Gutierrez, Jorge

Gómez-Serranillos, M. Pilar

Gominho, Jorge

Gonçalves, Catarina

Gonçalves, Lídia

Gonda, Sandor

Gong, Jirui

Gong, Rui

Gong, Seung Pyo

Gonzales, Eric B.

Gonzalez Halphen, Diego

González Laredo, Rubén F.

González Maglio, Daniel H.

Gonzalez Rosa, Juan Manuel

González, Florenci V.

Gonzalez, Zoilo

González-Díaz, Humbert

González-Díaz, Humberto

Gonzalez-Fernandez, Tomas

Gonzalez-Navarro, Herminia

González-Reimers, Emilio

Gonzalez-Stuart, Armando Enrique

Gooley, Paul

Gophna, Uri

Goreham, Renee

Goto, Akiteru

Göttert, Thomas

Götz, Klaus-Peter

Goumans, Marie-José

Govaere, Olivier

Gozal, Evelyne

Grabacka, Maja

Grabarczyk, Małgorzata 
Grabsztunowicz, Magda

Graczyk-Jarzynka, Agnieszka

Graether, Steffen

Graeve, Lutz

Graille, Marc

Grandi, Guido

Grandjean, Jan G.

Granja, Pedro L.

Granja, Tiago

Grant, Melissa

Graslund, Torbjorn

Grasso, Gianvito

Grativol, Clicia

Grattan, Lynn M.

Grayfer, Leon

Grayson, Dennis R.

Graziano, Guella

Greening, David

Gregáň, Juraj

Gregory, Karen J

Greive, Sandra

Gribskov, Michael

Griffiths, William

Grigorenko, Elena L.

Grimm, Marcus O. W.

Griñán-Ferré, Christian

Grishin, Alexander V.

Grocholewicz, Katarzyna

Gromadzinska, Jolanta

Grones, Peter

Groschner, Klaus

Groveman, Bradley

Groves, Matthew

Gruber, Christian

Grubić Kezele, Tanja

Grudnik, Przemyslaw

Grudzien-Nogalska, Ewa

Grunt, Thomas

Grusch, Michael

Grzmil, Paweł

$\mathrm{Gu}$, Wenyi

Gude, Natalie

Gueguen, Naïg

Guevara González, Ramón

Guharoy, Mainak

Guiard, Bruno

Guibert, Christelle

Guillén-Sánchez, Dominico A

Guix, Francesc Xavier

Gulati, Gaurav

Güldiken, Nurdan
Gulei, Diana

Gullberg, Donald

Gullon, Beatriz

Gullon, Betatriz

Gunasekera, Sunithi

Guo, Xin

Guo, Zheng

Gupta, Rishein

Gupta, Suprit

Gurrapu, Sreeharsha

Gurskaya, Nadya G.

Gursky, Jan

Gushchin, Ivan

Gushina, Irina

Gussago, Cristina

Gutiérrez, Laura

Gutowska, Izabela

Guzman, Fanny

Gyöngyösi, Mariann

Gyurcsik, Bela

Ha, Jung-Heun

Haas, Nikolaus

Hadži, San

Hafizi, Sina

Hagelueken, Gregor

Hagihara, Masaki

Hahm, Eunsil

Haitin, Yoni

Hakkinen, Lari

Hála, Michal

Halaban, Ruth

Halder, Amit Kumar

Haley, John D.

Hall, David H.

Hallett, Maurice B.

Hallmann, Ewelina

Hallsworth, J.E.

Halmosi, Róbert

Hamamoto, Ryuji

Hamblin, Michael

Hamdani, Nazha

Hamden, Khaled

Hammers, Christoph M.

Hammond, Gerry

Hamson, Dwayne

Han, Xiaobin

Hanafy, Nemany A. N.

Hanania, Michel

Handelsman, David

Hannun, Yusuf

Hano, Christophe 
Hanschen, Franziska

Hansíková, Hana

Hanski, Leena

Hanson, Julien

Hao, Jiaqing

Hao, Mingang

Hao, Nanjing

Happel, Christine M.

Hara, Akira

Hardman-Smart, Jonathan

Hardtke-Wolenski, Matthias

Harijan, Rajesh K

Harm, Stephan

Haro, Diego

Harper, James

Harpsøe, Kasper

Harris, Danni

Harrison, Freya

Harrison, Paul H. M.

Harrop, Richard

Hartman, Mariusz

Hartman, Matthew C.T.

Harvey, Peta

Hasanzadeh Kafshgari, Morteza

Haseltine, Cynthia

Hashemolhosseini, Said

Hashimi, Hassan

Hashimoto, Makoto

Hashimoto, Masaru

Hassell Jr., James E.

Hastings, Philip J.

Hatanaka, Tomomi

Hatano, Akihiko

Hatano, Taku

Hattori, Mitsuharu

Haubruck, Patrick

Haupt, Larisa M.

Hawinkels, Lukas

Hawkins, Michelle

Hawrył, Anna M.

Hayashi, Mariko Kato

Hayashi, Mikio

Hayashi, Mirian Akemi Furuie

Hayen, Heiko

He, Ming Fang

Heath, George

Heaton, Steven M.

Hećimović, Silva Katuŝić

Heddleston, John

Hedfalk, Kristina

Heiker, John T
Heinbockel, Thomas

Heine, Thomas

Héja, László

Helbert, William

Hendrickson, Tamara

Henen, Morkos

Hengesbach, Martin

Henkel, Janin

Heras Benito, Manuel

Heras, Francisco

Herde, Marco

Heredia-Guerrero, José Alejandro

Hering, Thomas

Hermenean, Anca

Hermida Prieto, Manuel

Hernández, Eliud Avier

Hernandez, Jean-François

Hernandez-Alonso, Pablo

Hernández-Cifre, José Ginés

Hernandez-Olmos, Victor

Héroux, Paul

Herrera, Federico

Hertzler, Philip L

Herzig, Volker

Hetényi, Csaba

Hetherington, Sandy

Hettiaratchi, Marian

Heuertz, Rita M.

Heyduk, Tomasz

Heyer, Wolf-Dietrich

Hibbitts, Alan

Hiebert, Paul

Hieronymus, Thomas

Higgins, Khadine

Hilhorst, Marc

Hinney, Barbara

Hinton, Deborah

Hirasawa, Noriyasu

Hiroaki, Hidekazu

Hirohata, Satoshi

Hirota, Kiichi

Hirotsu, Naoki

Hissa, Barbara

Hitchings, Matthew

Hjorth, Marit

Hlavaty, Juraj

Hluska, Tomáš

Ho, Eric $S$.

Ho, Vincent

Hocart, Charles

Hochstrasser, Mark 
Hodgson, David R. W.

Hoebeke, Johan

Hoelzl, Franz

Hofer, Anders

Hoff, Paula

Hoffman, Brad G.

Hoffman, Charles S.

Hoffmann, Lucia Vieira

Hofmann, Kay

Hohenegger, Martin

Hohmann, Miriam S. N.

Holdener, Bernadette C.

Holeček, Milan

Holefors, Anna

Holien, Jessica

Holien, Toril

Holland, Olivia

Holler, Eggehard

Holmes, Neil

Holt, Christine E.

Holzhauser, Thomas

Homma, Keiichi

Hondermarck, Hubert

Honek, John

Hong, Jeong Hee

Hong, Yan

Hooda, Jagmohan

Hoover, Timothy R.

Horakova, Jana

Hori, Mika

Horisawa, Sakae

Horman, Sandrine

Hornedo-Ortega, Ruth

Horowitz, Mia

Horvath, Dragos

Horwich, Arthur L.

Horwitz, Benjamin

Horzmann, Katharine

Hoshino, Masaru

Hosoyamada, Makoto

Hosui, Atsushi

Hour, Tzyh-Chyuan

Houry, Walid A.

Houston, Kevin D.

Howell, Brian

Hrenar, Tomica

Hsia, Shih-Min

Hsiao, Po-Jen

Hsieh, Feng-Chia

Hsieh, Tusty-Juan

Hsieh, Yves
Hsu, Pang-Hung

$\mathrm{Hu}$, Chang-min

$\mathrm{Hu}$, Chenlin

Huang, Dorothy Yu

Huang, Genin Gary

Huang, Hao

Huang, Hui-Chi

Huang, Jijun

Huang, Jin

Huang, Jui-Yen

Huang, Kuang-Tzu

Huang, Ming

Huang, MingQing

Huang, Shih-Yi

Huang, Stanley

Huang, Tze-Sing

Huang, Wei-Chien

Huang, Xudong

Huang, Yu-Chuen

Huda, Nazmul

Hudson, James

Huerta, Miguel

Hueso, Miguel

Huet, Sebastien

Hugelshofer, Michael

Hull, J.Joe

Humphreys, David

Humphries, Brock

Hung, Chin-Chuan

Hunt, Pamela

Huq, Md. Amdadul

Hur, Su-Mi

Hurlburt, Barry K.

Hurtado-Guerrero, Ramon

Husnjak, Koraljka

Hussein, Ahmed

Hutchins, Andrew P

Hutmacher, Dietmar

Hwang, Jae Sung

Hwang, Ming-Jing

Hwangbo, Cheol

Hyink, Deborah Pinson

Hyldegaard, Ole

Hynes, Michael F.

Hyun, Tae Kyung

Hyvönen, Marko

I. Kalinin, Vladimir

Iaccino, Enrico

Iannotti, Fabio Arturo

Ianora, Adrianna

Iasevoli, Felice 


\author{
Ibrahim, Ahmed \\ Iconomidou, Vassiliki \\ Idili, Andrea \\ Idris, Adi \\ Ientile, Riccardo \\ Ierardi, Enzo \\ Igarashi, Kazuei \\ Igual, Marta \\ Ihmels, Heiko \\ Iida, Kei \\ Ikeh, Mélanie \\ Ilari, Andrea \\ Iliakis, George \\ Ilies, Marc A. \\ Imada, Katsumi \\ Imai, Kenichi \\ Imai, Toshio \\ Imamoto, Yasushi \\ Imbimbo, Bruno \\ Imperatore, Concetta \\ Inaba, Hiroshi \\ Indiveri, Cesare \\ Inga, Alberto \\ Ingle, Shakti \\ Inoue, Atsuko \\ Inserte, Javier \\ Inta, Dragos \\ Inui, Masayuki \\ Inuzuka, Hiroyuki \\ Ippoliti, Rodolfo \\ Iqbal, Sumaiya \\ Iram, Surtaj \\ Iranzo, Olga \\ Irbäck, Anders \\ Irmisch, Sandra \\ Isaac, Vera Lucia Borges \\ Isaacs, Mark \\ Ishibashi, Nobuyuki \\ Ishida-Yamamoto, Akemi \\ Ishii, Isao \\ Ishii, Tetsuro \\ Ishizaki, Yasuki \\ Ishmael, Jane E. \\ Iskierko, Zofia \\ Iskra, Maria \\ Isola, Gaetano \\ Isom, Lori \\ Itabashi, Yutaka \\ Ito, Kazuo \\ Ito, Yuji \\ Ivanov, Andrey
}

Ivanova, Juliana M.

Iwamoto, Masahiro

Iwamoto, Naoki

Iwaniec, Urszula

Iwasaki, Kengo

Iwasaki, Takashi

Iwata, Hisataka

Iwata-Reuyl, Dirk

Iyer, Shilpa

Izawa, Kazuhiro P.

Izawa, Tetsuya

Izzo, Luana

Jachimska, Barbara

Jacobs, Hugues

Jacobsen, Kristian Mark

Jager, Martine J

Jaguva Vasudevan, Ananda Ayyappan

Jain, Ankur

Jain, Anshika

Jakas, Andreja

Jakob, Roman P.

Jakse, Jernej

Jakubík, Jan

Jakubowski, Witold

James, Nicholas G.

Janda, Elzbieta

Janda, Tibor

Jangampalli Adi, Pradeepkiran

Jankovic, Brankica

Jantas, Danuta

Jappe, Uta

Jaquet, Kornelia

Jaramillo-Flores, Maria

Jarosz-Wilkołazka, Anna

Järvinen, Tero A. H.

Jasiulionis, Miriam G.

Jaumot, Joaquim

Jayaraman, Shobini

Jaźwińska, Anna

Jee, Shiouhwa

Jelińska, Anna

Jena, Prasant Kumar

Jeng, Anna

Jennane, Rachid

Jensen, Ingvill

Jeon, Oju

Jeon, Sookyoung

Jeon, Young Ho

Jeong, Byeong-ryool

Jeong, Byung-Hoon

Jia, Zongchao 


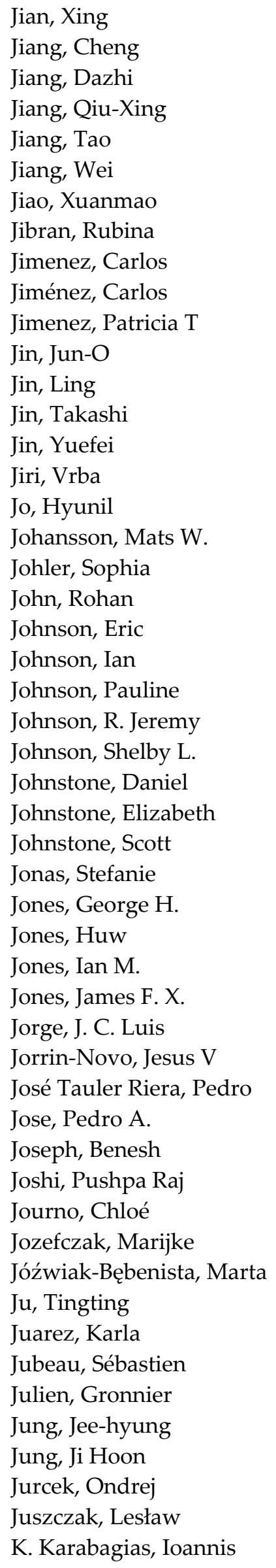

Kabelác, Martin

Kabil, Omer

Kabisch, Stefan

Kacaniova, Miroslava

Kačániová, Miroslava

Kaczanowski, Szymon

Kaczor, Jan Jacek

Kaczorowski, Tadeusz

Kaddes, Amine

Kadlubowski, Slawomir

Kafarski, Paweł

Kafel, Alina

Kagawa, Natsuko

Kai, Lei

Kajtár, Béla

Kalesh, Karunakaran

Kaliňáková, Barbora

Kaller, Markus

Kalogiouri, Natasa

Kalueff, Allan

Kamal, Fadia Ali

Kamari, Yehuda

Kamei, Yasutomi

Kaminski, Kamil

Kamiya, Mitsunobu

Kamzolova, Svetlana

Kanakkanthara, Arun

Kanata, Eirini

Kanda, Naoko

Kandasamy, Richard Kumaran

Kang, Byung-jae

Kang, CongBao

Kang, Hee-Gyoo

Kang, Hunseung

Kang, Kyuho

Kang, Seungwoo

Kang, Tae-Hong

Kang, Young-Hee

Kano, Hideaki

Kaplan, Barbara

Karcz, Dariusz

Karczmarzyk, Zbigniew

Kardos, Julianna

Kari, Lila

Karnati, Konda Reddy

Karpinski, Tomasz M.

Karunakaran, Suneesh

Kashiwagi, Shinichiro

Kaškonienè, Vilma

Kasuga, Kensaku

Katada, Kazuhiro 
Kataoka, Naoyuki

Kato, Takayuki

Katsiougiannis, Stergios

Katsyuba, Elena

Kawahara, Masahiro

Kawashima, Hideki

Kawauchi, Keiko

Kawczak, Piotr

Kawiak, Anna

Kayisli, Umit

Ke, Hengming

Ke, Liang-Yin

Kędziora, Anna

Keefover-Ring, Ken

Keinänen, Marja

Kejík, Zdeněk

Keller, Lance

Kellner, Stefanie

Kelly, Gregory M.

Kelly, Vincent

Kelts, Jessica

Kemp, Michael G.

Kenney, Maria Cristina

Kenny, Nathan James

Kerscher, Oliver

Ketkar, Amit

Kezic, Sanja

Khalifa, Shaden

Khalil-Ur-Rehman, Muhammad

Khan, Hamidullah

Khan, Irfan

Khan, Naeem

Khan, Sajid

Khan, Tanveer

Khananshvili, Daniel

Khashayar, Patricia

Khathi, Andile

Khatib, Firas

Khatib, Soliman

Khazaei, Hamid

Khedr, Mohammed A.

Khobta, Andriy

Khodarahmi, Reza

Kholmukhamedov, Andaleb

Kholová, Ivana

Khung, Yit Lung

Khurana, Namrata

Kieber-Emmons, Thomas

Kieffer, Nicolas

Kieliszek, Marek

Kierzek, Ryszard
Kiezun, Marta

Kikuchi, Haruhisa

Kikuchi, Kiyoshi

Kim, Bonglee

Kim, Byung-Soo

Kim, Chang-Eop

Kim, Chu-Young

Kim, Dohoon

Kim, Eun-Hee

Kim, Ha Hyung

Kim, Hak Jun

Kim, Hangun

Kim, Hoon

Kim, Hwan Keun

Kim, Hye Kyong

Kim, InKyeom

Kim, Jeong Beom

Kim, Jeung Gon

Kim, Ji-Hun

Kim, Jin-Kyung

Kim, Jong-Sang

Kim, Jung-Hwan

Kim, Junil

Kim, Ki-Hong

Kim, Kiyoung

Kim, Kyeong-Man

Kim, Kyoungtae

Kim, Kyung Jin

Kim, Mi-hyun

Kim, Min-Sik

Kim, Seung Wook

Kim, Soochong

Kim, T. Doohun

Kim, Yongsoo

Kim, Yoon Ki

Kim, Young Jun

Kim, Yun Kyung

Kimbaris, Athanasios

Kimura, Haruhide

Kimura, Hideto

Kimura, Seisuke

Kimura, Takefumi

Kimura, Tsuyoshi

King, Benjamin L.

Kinghorn, Kerri J.

Kinkorová, Judita

Kirberger, Michael

Kirby, Elizabeth D.

Kirchmaier, Ann

Kirchmair, Johannes

Kirimura, Kohtaro 
Kirpekar, Finn

Kirsch, Thorsten

Kiss, Robert

Kita, Toshihiro

Kitagawa, Kyoko

Kitchen, Philip

Kitsiouli, EIRINI

Kitzmüller, Claudia

Kizaka-Kondoh, Shinae

Kjaergaard, Magnus

Klatte-Schulz, Franka

Klein, Andrés D.

Klein-Júnior, Luiz Carlos

Klimontov, Vadim

Klochkov, S. G.

Klocko, Amy L.

Kloczkowski, Andrzej

Klöhn, Peter

Kluckova, Katarina

Knaus, Ulla G.

Knez, Jure

Knoche, Moritz

Knutsen, Erik

Kober, Kord

Kocerha, Jannet

Kocica, Mladen J.

Kociok, Norbert

Kodali, Maheedhar

Kodidela, Sunitha

Koelmel, Jeremy

Koenen, Rory

Koeppe, Roger E.

Koga, Fumitaka

Kogut, Michael H.

Koh, Cho Yeow

Kohout, Susy

Koike, Haruki

Kojima, Seiji

Kokoska, Ladislav

Kokotos, George

Koksharova, Olga A.

Kolář, Michal

Kolch, Walter

Kolesińka, Beata

Kolinski, Andrzej

Koltai, Hinanit

Komar, Anton

Komosinska-Vassev, Katarzyna

Konc, Janez

Kondakova, Irina

Kong Thoo Lin, Paul
Kong, Jiming

König, Gerhard

Konopelski, Piotr

Konstantinidis, Theocharis G.

Konstantinov, Spiro M.

Konto-Ghiorghi, Yoan

Kopečny, David

Kopsahelis, Nikolaos

Korac, Aleksandra

Korányi, Tamás

Korczeniewska, Olga

Korfiatis, Nikolaos

Korol, Sergiy

Kosakowska, Olga

Kosova, Klara

Kostareva, Anna

Kőszegi, Tamás

Kothapalli, Kumar SD

Kotloski, Robert

Kottakota, Chandrasekhar

Kottom, Theodore J.

Koufaki, Maria

Koulen, Peter

Koupenova, Milka

Kourkoutas, Ioannis

Kouroupis, Dimitrios

Koussis, Konstantinos

Koutelidakis, Antonios E.

Koval, Michael

Kovalenko, Andriy

Kovermann, Michael

Kovoor, Abraham

Koyama, Nobuhiro

Koyama, Tomotsugu

Kozakiewicz, Anna

Koziorowska-Gilun, Magdalena

Kozlakidis, Zisis

Kozlova, Elena

Kraeuter, Ann Katrin

Krag, Thomas $\mathrm{O}$

Krammer, Eva-Maria

Krasheninina, Olga A.

Krashias, George

Krásný, Libor

Krause, John R

Krautkramer, Kimberly A.

Krawczyk, Konrad

Kretz, Markus

Krga, Irena

Kristian, Tibor

Krokidis, Marios 
Krumova, Sashka

Kruszynski, Rafal

Krzepiłko, Anna

Krzyściak, Wirginia

Krzystanek, Marek

$\mathrm{Ku}$, Seung-Yup

Kuan, Yu-Hsiang

Kuban-Jankowska, Alicja

Kubatka, Peter

Kubíková, Lubica

Kubinova, Sarka

Kubo, Tai

Kubo, Takanori

Kubori, Tomoko

Kuča, Kamil

Kucharska, Karolina

Kucheryavykh, Lilia Y.

Kudryashova, Elena V.

Kudryavtsev, Denis S.

Kufareva, Irina

Kufel, Joanna

Kujawska, Małgorzata

Kuksa, Pavel P.

Kukula-Koch, Wirginia

Kulbacka, Julita

Kulkarni, Shashank

Kumar Mishra, Vivek

Kumar, Akhilesh

Kumar, Dhirendra

Kumar, Gokhlesh

Kumar, Narender

Kumar, Pradeep

Kumar, Rajeev

Kumar, Ujendra

Kumar, Vijay

Kume, Kensuke

Kunjukunju, Sangeetha

Kunoh, Tatsuki

Kunsági-Máté, Sándor

Kuo, Jen-Min

Kuo, Ming-Tse

Kurakula, Kondababu

Kuramata, Masato

Kurgan, Lukasz

Kurien, Biji T.

Kurtenbach, Stefan

Kusaczuk, Magdalena

Kusamori, Kosuke

Kushiro, Tetsuo

Kusters, Ilja

Kusumanchi, Praveen
Kusunose, Kenya

Kuter, Katarzyna

Kutikhin, Anton G.

Kutner, Andrzej

Kutryb-Zają, Barbara

Kuwahara, Tomoki

Kvasnica, Miroslav

Kwak, Brenda

Kwok, Henry Hang Fai

Kwon, Inchan

Kwong, Jennifer Q.

Kyei, George B

La Rosa, Carmelo

Labilloy, Anatalia

Laboisse, Christian L.

Labrador, María

Labruère, Raphaël

Lacadena, Javier

Lacatusu, Cristina Mihaela

Lachenmeier, Dirk

Lachowiec, Jennifer

Lacina, Lukas

Lacko, Andras

Lacruz, Rodrigo

Lafite, Pierre

Lafond, Mickaël

Lafont, Elodie

Lafontaine, Denis

Laforenza, Umberto

Laganà, Antonio Simone

Lagares, Alfonso

Lagares, David

Lagerstedt, Jens O.

Lagos, Leidy

Laguna, Ariadna

Lagunas, Beatriz

Lahiri, Tanaya

Lalas, Stavros

Lam, Sio-Hong

Lambeau, Gerard

Lambrev, Petar H.

Lamichhane, Rajan

Lampe, Paul

Lamy, Elsa

Lancel, Steve

Landi, Silvia

Landreau, Anne

Landrieu, Isabelle

Landucci, Elisa

Lanekoff, Ingela

Langdon, Simon 
Langella, Emma

Lanza, Giuseppe

Laradi, Sandrine

Lario, Luciana Daniela

Laroche, Céline

LaRue, Clayton $\mathrm{T}$.

Lasonder, Edwin

Lasorsa, Francesco Massimo

Latek, Dorota

Lattanzi, Simona

Lattanzio, Rossano

Lau-Cam, Cesar A.

Laurini, Erik

Lauro, Davide

Lavra, Luca

Law, Ruby

Ławicki, Sławomir ł.

Lawrence, Michael

Lazarus, Maja

Le Hir, Hervé

Le, Nguyen Quoc Khanh

Leadlay, Peter

Leaver, Michael

Lebaron, Richard

Leblond, Jeffrey

Lebrun, Marc-Henri

Lederkremer, Gerardo Z.

Lee, Bong Ho

Lee, Bor-Shiunn

Lee, Byoung Dae

Lee, Chang Hoon

Lee, Chia-Hwa

Lee, Dae-Sung

Lee, Eun Seong

Lee, Gabsang

Lee, Gun Woo

Lee, Hee Seung

Lee, I-Ta

Lee, Jaemin

Lee, Jintae

Lee, Kuo Hao

Lee, Kwanuk

Lee, Man Ryul

Lee, Mi Kyeong

Lee, Min Young

Lee, Sang Gil

Lee, Shao-Chen

Lee, Sukmook

Lee, Sung-Hyen

Lee, Wei-Chieh

Lee, Yen Chien
Lee, Yeon Sun

Lee, Younbok

Lee, Yu-Hsuan

Leem, Jaechan

Leeper, Thomas

Lees, Watson J.

Leffler, Hakon

Legembre, Patrick

Legrand, Fanny

Lehotsky, Jan

Lemasters, John J.

Lemli, Beata

Lemma, Enrico

Lenaghan, Scott

Leng, Roger

Leng, Siyang

Leon, Francisco

Leonardi, Salvatore

Leondaritis, George

Leppik, Liudmila

Lerma, Claudia

Les, Francisco

Lesgards, Jean-François

Leslie, Andrew G.W.

Lesnefsky, Edward J.

Lešnik, Samo

Lesyk, Roman B.

Letek, Michal

Leu, Steve

Leung, Yu min

Levi, Giovanni

Levick, Scott

Levy, Galit Katarivas

Lewczuk, Bogdan

Lewis, Aurélia E.

Lewis, Dale E.A.

Lewis, Gladius

Leybaert, Luc

Leychenko, Elena

$\mathrm{Li}$, Chenshuang

Li, Chien-Feng

Li, Chunyuan

Li, Guohui

Li, Haobo

$\mathrm{Li}, \mathrm{He}$

Li, Jiao Jiao

$\mathrm{Li}$, Jibin

LI, Jie

Li, Junhao

Li, Kuo-Bin

Li, Lianbo 


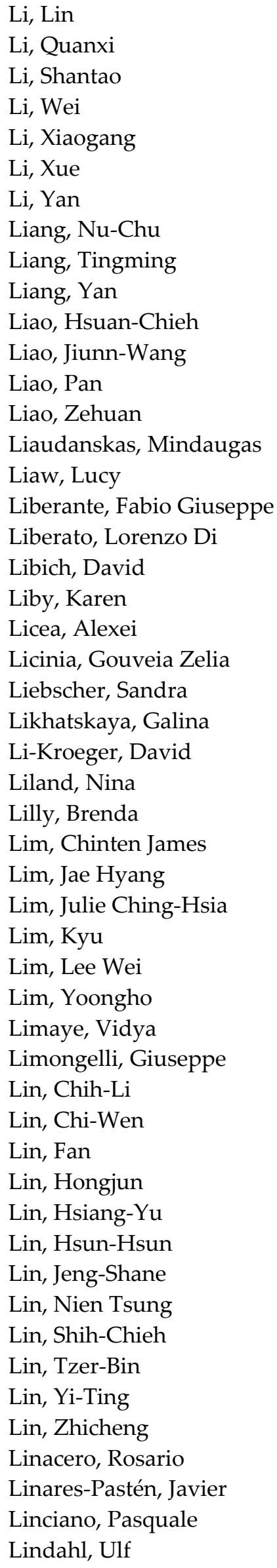

Linder, Benedikt

Linder, Stig

Lindsey-Boltz, Laura A.

Linsenmeier, Robert A.

Lionetti, Vincenzo

Liou, Jr-Jiun

Lipińska, Barbara

Lipscomb, Michael W.

Lisi, Lucia

Lisuzzo, Lorenzo

Liszkowska, Joanna

Liu, Bing-Lan

Liu, Chaoxing

Liu, Chunming

Liu, Fuguo

Liu, Gang

Liu, Guanglei

Liu, Hai-yang

Liu, Hongxiang

Liu, Junli

Liu, Ling

Liu, Mingxian

Liu, Tao

Liu, Wei

Liu, Xianglei

Liu, Yonggang

Liu, Yung-Chuan

Liu, Zhiping

LiWang, Patricia

Liwo, Adam

Llama-Palacios, Arancha

Llanes, Alejandro

Llavero, Francisco

Locascio, Annamaria

Locatelli, Marcello

Lock, Antonia

Loeffler, Christopher R.

Logan, Derek

Loghin, Felicia

Loh, Stewart

Loid, Josef

Loinard, Céline

Lombardi, Tommaso

Lombardi, Vincent C.

Lonardo, Amedeo

Long, Yuchen

Longley, Dan

Lonni, Audrey A. Stinghen Garcia

Lopes, Coeli M.B.

Lopes, Ricardo Jorge

López Fernández, Hugo 
López Martínez, Juan

Lopez, César A.

López, Kelly Johana Figueroa

Lopez, Victor

López-Camarillo, César

Lopez-Canul, Martha

López-Cebral, Rita

López-Hernández, Francisco J.

Lopez-Martin, Manuel

Lopez-Soto, Alejandro

Loring, Ralph H.

Losurdo, Giuseppe

Lothier, Jérémy

Louis, Corentin

Loureiro, Joana A.

Lourenço, Ana

Löw, Péter

Loza-Mejía, Marco A.

$\mathrm{Lu}$, Jeng-Wei

Lu, Wan-Jung

Lubin-Germain, Nadège

Lucafò, Marianna

Lucas, Andrew T.

Luce-Fedrow, Alison

Lucentini, Livia

Luchinat, Enrico

Ludidi, Ndiko

Ludwikõw, Agnieszka

Lugao, Ademar

Lui, Edmund Man King

Luigi, Palmieri

Luisa, Benussi

Łukasz Konefał, Rafał

Lund, Pete

Lundström, Claes

Luongo, Livio

Lupattelli, Paolo

Lutfalla, Georges

Luthra, Priya

Luwor, Rodney

Lux, Thomas

Lux-Battistelli, Christine

Lv, Huixiong

Łyczko, Jacek

Lye, Gary

Lymperopoulos, Anastasios

Lynch, Marina

Lyons, Shawn

Lyukmanova, Ekaterina N.

M. Aizpurua, Jesús

Ma, Chengbang
Ma, Guojia

Ma, Jiyan

Ma, Wei

Macauley, Matthew S.

Maccallini, Cristina

Macchi, Chiara

MacDiarmid, Colin W.

Macek Jílková, Zuzana

Macnaughten, Meghan

Maddelein, Marie-Lise

Madine, Jill

Madine, Jillian

Madrid, Alejandro

Madureira, Patricia

Maechler, Pierre

Maeng, Sung Ho

Maestro, Beatriz

Maffei, Angelo

Maftah, Abderrahman

Mafu, Sibongile

Magarlamov, Timur Yu.

Maggi, Davide

Magistrato, Alessandra

Magne, David

Magnus, Marcin

Magyar, Csaba

Mahavadi, Sunila

Mahmassani, Ziad S.

Mahmoud, Ayman

Mains, Richard E.

Maisch, Bernhard

Maity, Shuvadeep

Majtan, Tomas

Majumdar, Susruta

Makarevich, Pavel

Makarova, Kira S

Makhutova, Olesia

Makoto, Hirayama

Makowska, Krystyna

Maksymiuk, Andrew W.

Malapelle, Umberto

Malarkannan, Subramaniam

Malejko, Julita

Małgorzata, Kowalska

Malinowska, Elzbieta

Mallela, Shamroop Kumar

Mallipeddi, Prema Latha

Malone, Jacob

Malys, Naglis

Mambu, Lengo

Mammadova-Bach, Elmina 
Mandraffino, Giuseppe

Mangiagalli, Marco

Mangino, Giorgio

Mangogna, Alessandro

Mangoni, Maria Luisa

Mani, Alireza

Maniati, Eleni

Mankin, Alexander

Mann, Francis

Männer, Jörg

Mansour, Salah

Mansuri, Shahid

Manta, Stella

Mantere, Outi

Manzo-Merino, Joaquin

Manzoni, Olivier J.

Mao, Xiaobo

Mao, Xuming

Marabello, Domenica

Maraia, Richard

Marasek-Ciolakowska, Agnieszka

Marazuela, Monica

Marc, Gabriel

Marchand, Christophe

Marchetti, Dario

Marchev, Andrey

Marchini, Cristina

Marcinkowska, Ewa

Marco-Contelles, Jose Luis

Marczyk, Michal

Maréchal, Alexandre

Maresca, Marc

Margină, Denisa

Margiotta, Azzurra

Margis, Rogerio

Marí, Montserrat

Maria Carmela, Bonaccorsi Di Patti

Marietta, Fodor

Marinello, Francesco

Marino, Angela

Marino, Valerio

Markova-Car, Elitza P.

Markowicz-Piasecka, Magdalena

Markowska-Daniel, Iwona

Markwardt, Fritz

Marlowe, Timothy

Marmagne, Anne

Maroni, Paola

Maroteaux, Luc

Marques, Alexandra P.

Marques, Mariana
Marquez, Edgar

Marrs, James A.

Marsche, Gunther

Marshall, Craig

Martelli, Francesco

Martens, Gerard

Martí, Sergio

Martin, Andreas

Martin, Andrew C.R.

Martin, Franck

Martín, Juan Francisco

Martin, Pamela

Martinez, Agustin

Martinez, Ana

Martínez, Constantino

Martínez, Sonia

Martínez-Banaclocha, Marcos Arturo

Martínez-Campa, Carlos

Martínez-Force, E.

Martinez-Gonzalez, Luis J.

Martínez-Martínez, Irene

Martínez-Salas, Encarnación

Martínez-Yamout, Maria A.

Martínková, Ludmila

Martín-Montalvo, Alejandro

Martinou, Jean-Claude

Martins, Natalia

Martins, Pedro M.

Martín-Ventura, José Luis

Maruca, Annalisa

Maruška, Audrius

Marutescu, Luminita Gabriela

Marverti, Gaetano

Mary, Didier

Massa, Annamaria

Massaro, Marina

Mast, Yvonne

Mastanjević, Kristina

Mastinu, Andrea

Mastoraki, Aikaterini

Mastracci, Teresa

Mastrangelo, Anna M.

Mastrotto, Francesca

Masuda, Kiyoshi

Masuyer, Geoffrey

Matafome, Paulo N.

Matás-Parra, Carmen

Mateo-Bonmatí, Eduardo

Matesic, Lidia

Mateus, Maria L.

Matichenkov, Vladimir V. 
Matilla, Miguel

Matkowski, Adam

Mato, Eugenia

Mato, Susana

Matsuda, Hisashi

Matsufuji, Hiroshi

Matsugaki, Aira

Matsukawa, Noriyuki

Matsumoto, Taro

Matsuura, Kazunori

Matsuzaki, Juntaro

Matsuzaki, Keiichi

Mattei, Benedetta

Mattei, César

Mattinen, Maija-Liisa

Mattingly, Raymond R.

Mattoo, Autar K.

Mattos, Carla

Matucci, Rosanna

Matulić, Maja

Matuszewska, Anna

Matwijczuk, Arkadiusz

Matysiak, Jan

Matysiak, Joanna

Matziari, Magdalini

Mauceri, Rodolfo

Mavon, Alain

Mavragani, Clio

Mavri, Janez

Mavrogonatou, Eleni

Mayan, Maria D.

Mayeda, Akila

Mayer, Thomas U.

Mayo, Juan Carlos

Mayo, Kevin

Mazerbourg, Sabine

Mazur, Marcelina

Mazzanti, Andrea

Mazzulli, Joe

Mccully, Kilmer S.

McDowell, Colleen M.

McGavin, Martin J.

McGuckin, Michael

McHugh, Kevin J.

McKay, Tina

McKinnie, Shaun

McKinstry, Karl Kai

Md Badrul, Alam

Mears, Jason

Meccariello, Rosaria

Medici, Serenella
Medina-Rodríguez, Eva M.

Medraño-Fernandez, Iria

Medvedev, Alexei

Meerson, Ari

Megyeri, Klara

Mehta, Gaurav A.

Meinander, Annika

Meiners, Silke

Meldolesi, Jacopo

Mellata, Melha

Memo, Maurizio

Menanteau-Ledouble, Simon

Mendez-Cuadro, Darío

Mendonca, Patricia

Mendoza, Artemio

Menegon, Andrea

MENEGUZZO, FRANCESCO

Menendez, Daniel

Meneses, Carlos

Menezes, Irwin Rose Alencar

Mennerich, Daniela

Merimi, Makram

Merino Peláez, Gracia

Mertens, Peter

Meškys, Rolandas

Meslet-Cladiere, Laurence

Messina, Samantha

Messner, Barbara

Meštrović, Tomislav

Metzinger, Laurent

Meyer, Thomas J.

Mézes, Miklós

Mezheyeuski, Artur

Mezquita, Cristóbal

Michaud, Philippe

Michlewski, Gracjan

Middleton, Adam

Midgley, Adam

Mignatti, Paolo

Miguel-Hidalgo, Jose Javier

Mika, Delphine

Mikolajczak, Przemyslaw

Mikros, Emmanuel

Milani, Adelaide

Milanovic, Desanka

Milcarek, Christine

Mileva, Milka

Millán-Linares, María Del Carmen

Miller, Charles

Millet, Óscar

Minato, Ken-ichiro 
Miniero, Daniela Valeria

Minucci, Sergio

Minutolo, Filippo

Miosge, Nicolai

Mir, Ricardo

Miranda, Cristobal

Miranda, Hugo

Mirkov, Ivana

Mishra, Awdhesh

Mishra, Geetesh K.

Mishra, Manish

Mishra, Nigam

Mishra, Pawan Kumar

Mishra, Sandeep Kumar

Miskulin, Maja

Mitchell A, Sullivan

Mitchell, Jennifer A.

Mitchell, Molly E.

Mitchell, Todd

Mitola, Stefania

Mitraki, Anna

Miwa, Julie M.

Miyakawa, Isamu

Miyamoto, Yoichi

Miyata, Yasuyoshi

Miyazaki, Ikuko

Miyoshi, Eiji

Miyoshi, Shin-ichi

Mizrahi, Boaz

Mizumoto, Shuji

Mladenović, Milan

Mo, Fei

Mobbili, Giovanna

Mocquet, Vincent

Mofrad, Mohammad

Mohamed, Azza H.

Mohamed, Junaith S.

Mohammed, Altaf

Moiseyev, Gennadiy

Moldovan, Bianca

Molina Salinas, Gloria María

Molina, Lázaro

Molinier, Jean

Mollen, Kevin P.

Molnár, Elek

Monari, Antonio

Mondal, Goutam

Mondal, Sudip

Mondal, Utpal Kumar

Mondeshki, Mihail

Monné, Magnus
Monsivais, Diana

Montag, Dirk

Montagne, Kevin

Montana, Angel M.

Monte Lara, $\mathrm{M}^{\mathrm{a}}$ Concepción

Monti, Maria Chiara

Montpetit, Ben

Moore, Ronald B

Moore, Stuart

Morak-Młodawska, Beata

Morales, Ludis

Morales-Morales, David

Morante, Koldo

Moreira, Daniel Carneiro

Moreira, Manuela M.

Moreira, Paula

Morello, Roy

Moreno Amador, María Lourdes

Moreno Gonzalez, Ines

Moreno, Esther

Moretti, Fabiola

Morgan-Bathke, Maria

Morgat, Clément

Morgunov, Igor

Moricz, Agnes

Moriishi, Kohji

Morikawa, Satoru

Morimoto, Yusuke V.

Morinobu, Akio

Morita, Tomotake

Moriya, Manabu

Moriyama, Mariko

Moriyama, Tatsuya

Morla, Shravan

Morleo, Manuela

Morley, Barbara J.

Moro, Enrico

Moro, Loredana

Morocho, Vladimir

Morozova-Roche, Ludmilla

Morris, Andrew

Morrison, Keith D.

Mortara, Lorenzo

Moseley, Hunter

Moses, Tessa

Moskalewicz, Tomasz

Moskot, Marta

Moss, Mark

Motin, Vladimir

Motohashi, Ken

Mott, Helen 
Motti, Maria Letizia

Mouga, Teresa

Mounien, Lourdes

Moya-Ramírez, Ignacio

Mravljak, Janez

Mrozek-Wilczkiewicz, Anna

$\mathrm{Mu}$, Yunxiang

Muench, Stephen

Mugisho, Odunayo

Mukaida, Naofumi

Mukhopadhyay, Suman

Mulenga, Albert

Müller, Christoph

Müller, Norbert

Müller-Taubenberger, Annette

Mulligan, Vikram K.

Mulloy, Barbara

Mulo, Paula

Mummidi, Srinivas

Mumoli, Nicola

Muñoz Caffarel, María

Muñoz, Francisco

Munteanu, Florentina-Daniela

Munusamy, Elango

Murakami, Hironobu

Murakami, Katsuhiko S.

Murakami, Makoto

Murakami, Masaaki

Muramoto, Koji

Murase, Sachiko

Murata, Shigeo

Murkin, Andrew

Muronetz, Vladimir

Muroya, Susumu

Murphy, Caroline S

Murray Stewart, Tracy

Murthy, Vinuthaa

Musalgaonkar, Sharmishtha

Muscara, Marcelo N.

Musco, Giovanna

Mustafa, Ahmed M.

Muszyński, Siemowit

Mutti, Luciano

Muyldermans, Serge

Myöhänen, Timo T.

Mythreye, Karthikeyan

$\mathrm{Na}$, Dong Hee

$\mathrm{Na}$, MinKyun

Naafs, Bernard

Nadăş, George Cosmin

Naderi, Mohammad
Nadiminty, Nagalakshmi

Nagafuchi, Yasuo

Nagaoka, Isao

Nagaraj, Vini

Nagendran, Jeevan

Naggi, Annamaria

Nagy, Veronika

Nagyova, Eva

Naim, Hassan

Nair, Sreenath

Najdekr, Lukáš

Nakagaki, Takehiro

Nakagawa, Pablo

Nakahata, Masaki

Nakajima, Kei

Nakamaru-Ogiso, Eiko

Nakashima, Souichi

Nakata, Rieko

Nakatani, Yosuke

Nakatsu, Kanji

Nakazawa, Yasumoto

Nalepa, Irena

Namasivayam, Vigneshwaran

Namkung, Wan

Nance, Elizabeth

Nanda, Sitansu Sekhar

Nanduri, Ravikanth

Nanoff, Christian

Narayanan, S. Priya

Nardo, Luca

Naro, Fabio

Narra, Hema Prasad

Naryzhny, Stanislav N

Nash, Michael A.

Nassir, Fatiha

Natalello, Antonino

Natalicchio, Annalisa

Nauli, Surya

Naumowicz, Monika

Navarro, Juan Antonio

Naya, Francisco

Nazar, Ross N.

Nazzaro, Filomena

Neeb, Antje

Neginskaya, Maria A.

Németh, Balázs

Nesmelova, Irina

Nesterkina, Mariia

Neubauer, Heidi

Neustupa, Jiri

Neu-Yilik, Gabriele 
Neves, João

Newman, David

Newton, Phillip

$\mathrm{Ng}$, Chen Siang

$\mathrm{Ng}$, Jason Liang Pin

$\mathrm{Ng}$, Roy Chun-Laam

Ngara, Rudo

Ngezahayo, Anaclet

Nguyen, Van Khanh

Nicaud, Jean-Marc

Nicolle, Remy

Nicosia, Aldo

Niculescu, Loredan

Niczyporyk, Jowita Samanta

Niederreither, Karen

Nieland, John Dirk Vestergaard

Nielsen, Sofie V.

Niemann, Hartmut $\mathrm{H}$.

Niero, Giovanni

Nikitovic, Dragana

Nikolaivits, Efstratios

Nikolich, Mikeljon

Nikolova, Maria

Nilsen, Hilde

Nilsson, Ake

Ninfali, Paolino

Nioi, Claudia

Nirala, Niraj K.

Nirmala, Jayaveeramuthu

Nirody, Jasmine A.

Nishi, Kosuke

Nishimiya, Yoshiyuki

Nishitsuji, Kazuchika

Nisticò, Paola

Nitulescu, George Mihai

Nociari, Marcelo

Nohe, Anja

Nomura, Masatoshi

Nonaka, Taichiro

Nonogaki, Katsunori

Nord, Ashley

Nordmark, Gunnel

Norihiko, Narita

Notarbartolo, Monica

Notarstefano, Valentina

Novak Kujundžić, Renata

Novinec, Marko

NR, Parine

Nucci, Nathaniel

Nunes, Alexandra

Nunez, Cesar
Nunzio, Vicario

Nurunnabi, Md

Nussinov, Ruth

Nuttall, Patricia A

Nycz, Jacek

Nyström, Alexander

O'Leary, Seán

Obeng, Esther A.

Oberemok, Volodymyr V.

Oberle, Doris F.

Obermayer, Benedikt

Ochi, Shinichiro

Ochieng, Josiah

Ochsenreither, Katrin

Odland, Jon

Oelschlaeger, Peter

Ofir, Rivka

Ogawa, Takahiro

Oh, Deog-Hwan

Oh, Jae-Min

Oh, Joonseok

Oh, Seung Ja

Ohki, Rieko

Ohko, Kentaro

Ohlenschläger, Oliver

Ohta, Kiminori

Ohta, Nobuo

Oishi, Kimihiko

Okada, Motohiro

Okae, Hiroaki

Okamura, Ryosuke

Okolicsanyi, Rachel K

Oku, Masahide

Okuda, Kazuhide

Olejar, Kenneth J.

Olennikov, Daniil

Oliva, Romina

Oliveira, Ana

Oliveira, Guedmiller S.

Oliveira, Sabrina

Oliviero, Francesca

Ollero, Mario

Olofsson, Malin

Olsen, Birgitte

Olsen, Lars Folke

Olson, Eric

Olson, Kenneth

Olsson, Stefan

Olszewska, Marta

Oltean, Sebastian

Oluwadare, Oluwatosin 
Omar, Syed Haris

Ondrias, Karol

Onduka, Toshimitsu

Ong, Qunxiang

Oniga, Ilioara

Oniga, Smaranda Dafina

Onoyama, Ichiro

Opaliński, Łukasz

Oprea, Corneliu Ioan

Oprică, Lăcrămioara

Ordaz-Ortiz, José Juan

Orenes Piñero, Esteban

Oriá, Reinaldo B.

Orian, Laura

Oriuchi, Noboru

Orlando, Giustino

Orłowski, Marek

Orrú, Christina

Ortega-Villaizan, Maria Del Mar

Osadnik, Tadeusz

Osmanagic-Myers, Selma

Osmulski, Pawel A.

Ostacolo, Carmine

Ostash, Bohdan

Ostoa-Saloma, Pedro

Ostrovsky, Olga

Ota, Motonori

Ott, Maria

Ougham, Helen

Oury, Bruno

Ovádi, Judit

Overmyer, Katherine

Oves-Costales, Daniel

Owen, Shawn

Ozaki, Toshinori

Ozawa, Shogo

Ozen, Mehmet

Ozimek, Ewa

Paavola, Jere

Pace, Loretta

Pacheco, Neith

Pacholski, Andreas

Paci, Maurizio

Paczesny, Jan

Paduszynska, Malgorzata

Pagès, Vincent

Paidi, Santosh Kumar

Painter, Heather

Pakhomova, Svetlana

Palage, Mariana

Palanisamy, Arulselvan
Palanisamy, Uma Devi

PALAVAI, SRIPAL REDDY

Palenzuela López, José Antonio

Palermo, Giulia

Palini, Simone

Pallàs Lliberia, Mercè

Palma, Miguel

Palmer, Alan

Palmeri, Rosa

Palomo, Valle

Palumaa, Peep

Palyulin, Vladimir

Pan, Cuiping

Pan, Jiayi

Pan, Pingyue

Panaro, Maria Antonietta

Panatala, Radhakrishnan

Pancsa, Rita

Panda, Rakhi

Pandey, Avinash Chandra

Pandey, Chandana

Pang, Myung-Geol

Pannek, Jürgen

Pantano, Lorena

Panzella, Lucia

Paoli, Paolo

Papaioannou, Emmanouil

Papathanasiou, Fokion

Papini, Alessio

Papp, Gábor

Parchi, Piero

Pardo, Fatima

Pardo, Marta

Parenti, Carmela

Parikesit, Arli Aditya

Paris, Juan

Parisi, Ludovica

Park, Daeho

Park, Edwards A.

Park, Jong Kook

Park, Seong-Cheol

Park, Wansu

Park, Woo Jae

Park, Yoonseong

Parkar, Shanthi G.

Parkkita, Seppo

Parmeggiani, Fabio

Parnetti, Lucilla

Parra, Margarita

Parrino, Vincenzo

Pârvu, Alina Elena 
Pascal, Steven M.

Pasciu, Valeria

Pashov, Anastas

Pasqualini, Francesco

Pasquinelli, Gianandrea

Pastores, Gregory M.

Pašukonienė, Vita

Patelski, Piotr

Patra, Jayanta Kumar

Patro-Małysza, Jolanta

Patterson, Jennifer

Paudel, Indira

Paul, Ananya

Paul, Sudip

Pautz, Andrea

Pavlik, Edward J.

Pavlin, Matic

Pawlik, Anna

Pearce, Margaret

Peart, Jason

Pec, Martin

Pecic, Stevan

Pecqueur, Claire

Pedemonte, Nicoletta

Pedersen, Per Amstrup

Pedeux, Rémy

Pedraza Chaverri, José

Pedrero, Maria

Pedroso, João Alfredo

Pegan, Scott D.

Peiser, Matthias

Peitzsch, Claudia

Peixoto, Paul

Pelagalli, Alessandra

Pellegrini, Marika

Pellegrino, Marsha

Pelzer, Elise

Pemp, Berthold

Peña Vega, Fernando

Peña-Espinoza, Miguel

Peña-Rodríguez, LM

Penas, Clara

Pence, Brandt

Peng, Robert $\mathrm{Y}$.

Peng, Wenjing

Peng, $\mathrm{Xu}$

Pentimalli, Francesca

Penzo, Marianna

Peptu, Cristian

Perdomo, Jose

Perego, Paola
Pereira, Cláudia

Pereira, Florbela

Pereira-Leite, Catarina

Perestrelo, Rosa Maria De Sá

Pérez - Amodio, Soledad

Pérez De Vega, María Jesús

Perez-Hernandez, Javier

Perez-Mendez, Oscar

Pergolizzi, Simona

Perinelli, Diego Romano

Perišić, Ognjen

Periyannan, Sambasivam

Perkins, Arden

Permyakov, Eugene

Pernisová, Markéta

Pernodet, Jean Luc

Pero, Raffaela

Perra, Andrea

Perreault, Melissa

Perricone, Carlo

Perrucci, Stefania

Pers, Yves-Marie

Perugino, Giuseppe

Peruzynska, Magdalena

Pestov, Dimitri

Petan, Toni

Petca, Răzvan

Peter, Emanuel Karl

Peters, Christian

Petersen, Robert B.

Petersen, Svea

Peterson, Julia

Peterson, Larryn

Peterson-Hiller, Amie

Petit, Patrice X.

Petrov, Alexey M.

Petter, Michaela

Pezzolesi, Laura

Pfeffer, Lawrence M.

Pfister, Astrid S.

Pfister, Barbara

Phelix, Clyde F.

Philibert, Danielle

Philpott, Caroline

Piațek, Rafał J.

Piato, Angelo

Piccialli, Gennaro

Piccionello, Antonio Palumbo

Piccoli, Marco

Pick, Elah

Pidoux, Guillaume 
Piedras, Pedro

Piehler, Jacob

Pieretti, Stefano

Pierri, Ciro Leonardo

Pierzchalski, Piotr

Piestrzyńska-Kajtoch, Agata

Pietropaolo, Adriana

Pievani, Alice

Pignitter, Marc

Pigossi, Suzane Cristina

Pilling, Darrell

Pineda, José R.

Pineda, Jose Ramon

Piñeiro, Angel

Pinelis, V. G.

Ping, Yueh-Hsin

Pinter, Tyler

Pinteus, Susete

Pinto, Sandra

Pintus, Francesca

Pintus, Gianfranco

Piomboni, Paola

Piotrowicz-Cieślak, Agnieszka

Piras, Anna Maria

Pires, Bruno R. B.

Pires, Carla

Pirola, Luciano

Pirooznia, Mehdi

Pishchalnikov, Roman Y.

Pissas, Georgios

Pistarà, Venerando

Pistocchi, Anna Silvia

Pitingolo, Gabriele

Pitts, Matthew

Piva, Terrence

Piya, Sarbottam

Pizoń, Magdalena

Pizzo, Elio

Plant, Leigh

Platta, Harald

Platts, James

Plaza-Zabala, Ainhoa

Plenis, Alina

Pleschka, Stephan

Plíhal, Ondřej

Pluta, Ryszard

Podlipnik, Črtomir

Podobnik, Marjetka

Poeggeler, Burkhard

Pohl, Ehmke

Poinsot, Verena
Pojo, Marta

Póka, Róbert

Polacek, Norbert

Polčic, Peter

Polhemus, David

Polit, Agnieszka

Pollastri, Gianluca

Pollice, Alessandra

Poloni, Tino Emanuele

Pomari, Elena

Pompilio, Giulio

Ponce-Vargas, Miguel

Ponnalagu, Devasena

Pooler, Darcy

Poon, Gregory

Pop, Horia F

Pope, Chad

Popescu, Mihaela R.

Popgeorgiev, Nikolay

Popielarska-Konieczna, Marzena

Poplawski, Tomasz

Porcari, Andréia De Melo

Porrini, Vanessa

Potanin, Andrei A.

Potočnik, Uroš

Potočnjak, Iva

Potrich, Cristina

Pottosin, Igor

Poulin, Remington

Poupot, Remy

Pourcet, Benoît

Pourzand, Charareh

Powell, Folami Lamoke

Powell, Gregory

Power, David

Powers, Rachel

Pracharova, Jitka

Pramanik, Avijit

Prasad, Ram

Prasanna, Pradeep

Praud, Christophe

Preiner, Darko

Preiss, Thomas

Prelipceanu, Marius

Prester, Ljerka

Preti, Delia

Priault, Muriel

Prieto-Garcia, Jose M.

Primožič, Ines

Prinz, William

Pritzker, Kenneth 
Pritzkow, Sandra

Procházková, Jiřina

Proctor, Spencer D.

Prudent, Julien

Prusty, Bhupesh

Psarras, Stelios

Psenicka, Martin

Psurski, Mateusz

Puglisi, Rita

Puig, Berta

Pulido, Rafael

Puniya, Bhanwar Lal

Puppo, Francesca

Püschel, Gerhard

Pyatibratov, Michael

Pyka, Alina

Qi, Feng

Qi, Junpeng

Qi, Phoebe X.

Qian, Chunqi

Qiao, Huanyu

Qimron, Udi

Qin, Ren Yi

Qneibi, Mohammad

Quan, Taihao

Quattrocelli, Mattia

Quinlan, Roy A.

Rabanal Anglada, Francesc

Rabbani, Gulam

Raccuia, Salvatore Antonino

Radeghieri, Annalisa

Rademaker, Gilles

Rader, Christoph

Radisavljevic, Ziv

Radko, Lidia

Radominska-Pandya, Anna

Radosavljević, Tatjana

Radosinska, Jana

Radosinski, Lukasz

Radulescu, Cristiana

Rafacho, Alex

Rafiq H Siddiqui, Mohammed

Ragavan, Mukundan

Ragg, Enzio

Raggi, Carla

Ragnini-Wilson, Antonella

Rahman, Azizur

Raimondi, Claudio

Raimondo, Domenico

Raimundo, Nuno

Raina, Satish
Rajagopal, Sudarshan

Rajakumar, Nagalingam

Rajarapu, Swapna Priya

Rajput, Akanksha

Rajput, Vishnu D.

Ramachandiran, Iyappan

Ramachandran, Anup

Ramalingam, Nagendran

Ramanadham, Sasanka

Ramirez, Giuseppe A.

Ramon Osman, Tasan

Ramos, Adrián M.

Rampanelli, Elena

Rando, Daniela

Rando, Maria Margherita

Rankin, Gary

Rao, Gundu H.R.

Raouche, Sana

Rappocciolo, Giovanna

Rashid, Mohammad Harun

Rashkov, Iliya

Rasinger, Josef Daniel

Raspor, Martin

Ratajewski, Marcin

Rathinasabapathy, Anandharajan

Rato, Ana Elisa

Rätsep, Matthew

Rau, Ileana

Ravaioli, Stefano

Ravalli, Silvia

Ravanidis, Stylianos

Ravera, Enrico

Ravid, Tommer

Rawel, Harshadrai

Ray, Supriyo

Raymond, Josette

Reardon, Patrick

Rébé, Cédric

Rebelo, Sandra

Rebuffat, Sylvie

Rechkoblit, Olga

Recinella, Lucia

Reddy, Sai T.

Reddy, Sakamuri

Reffuveille, Fany

Reimann, Regina R.

Reinhardt, Christoph

Reinke, Hans

Reipa, Vytas

Reis, Ana

Rejinold, Sanoj 
Remelli, Maurizio

Remenyik, Judit

Remmas, Nikolaos

Ren, Xiaoyuan

Ren, Yanrong

Renault, Nicolas

Requena, David

Requena, Jesus

Resendiz, Marino

Resnick, Andrew

Restani, Laura

Reszka, Edyta

Retamal, Mauricio

Reverter, Miri

Reyes-Batlle, María

Reymond, Jean-Louis

Reynoird, Nicolas

Rezaei-Ghaleh, Nasrollah

Rezvani, Khosrow

Rhazi, Larbi

Rhett, Joshua Matthew

Rho, Jung-Rae

Rhodes, Lyndsay V.

Rialland, Mickaël

Riaz, Adnan

Riazuddin, Saima

Ribaudo, Giovanni

Ribeiro Macedo, Sandra

Ribeiro, Apoena

Ribeiro, Laura

Ribot, Joan

Riccardi, Claudia

Ricci, Claudia

Riccò, Matteo

Richards, Joanne S.

Richardson, Bruce

Richardson, Lauren

Richter, Magdalena

Riddiford, Lynn M.

Rieger-Christ, Kimberly M

Rifler, Jean-Pierre

Riljak, Vladimír

Rise, Patrizia

Rispail, Nicolas

Rissiek, Björn

Rittmann, Simon

Rivera, Guillermo

Rivera-Monroy, Zuly Jenny

Rivera-Pérez, Crisalejandra

Rizzi Sanches, Elen

Rizzo, Carmen
Rizzuti, Bruno

Robaszkiewicz, Agnieszka

Robert, Quinn

Robertson, Janice

Robinson, Stacey L.

Robson, Matthew James

Rocha, Emily M.

Rocha, João

Rocha, Murilo R.

Rochais, Christophe

Rockenfeller, Patrick

Rodenbeck, Stacey Dineen

Rodrigo-Comino, Jesus

Rodrigues, Célia F.

Rodrigues, Eliana

Rodrigues, Joaquim Rui

Rodrigues, Márcio

Rodríguez González, Vicente

Rodriguez, Alvaro

Rodríguez, Manuel S.

Rodriguez-Esteban, Raul

Rodríguez-Pérez, Ana Isabel

Rodriguez-Vita, Juan

Roeters, Steven Joop

Rogasevskaia, Tatiana

Rogers, Kim

Rogers, Simon

Roh, Sangho

Roh, Taehyun

Rohde, John R.

Rolla, Simona

Rolland, Filip

Rolle, Luca

Romanelli, Maria Novella

Romano, Patrizia

Romanov, Georgii

Románszki, Loránd

Roncevic, Tomislav

Rong, Chan Kuan

Rosas Cárdenas, Flor De Fátima

Rosca, Ana Maria

Roseanu, Anca

Rosellò Catafau, Joan

Rosenberger, Thad A.

Rosenberry, Terrone

Rosencrantz, Ruben R.

Rosenzweig, Derek

Rosenzweig, Steven A.

Ross, Avena

Ross, Jen

Ross, Samir 
Rossi, Elisa

Rossignol, Julien

Rossing, Maria

Rosso, Natalia

Rossotti, Martin A.

Rostovtseva, Tatiana

Roszczenko-Jasinska, Paula

Roterman, Irena

Roth, Michael

Rothbauer, Mario

Rothbauer, Ulrich

Rottbauer, Wolfgang

Roucou, Xavier

Rouhier, Matthew F.

Rousset, Raphaël

Roy, Anuradha

Roy, Peter J.

Roy, Swapan Kumar

Rozhon, Wilfried

Ruben, Pereira

Rubio, Lourdes

Ruckdeschel, Klaus

Rudack, Till

Rüdiger, Stefan

Rudolph, Johannes

Ruelland, Eric

Ruf, Viktoria C.

Ruffinatti, Federico Alessandro

Ruhanya, Vurayai

Ruiz Mendez, Maria

Ruiz, Mario

Ruiz-Canela, Miguel

Ruiz-Gómez, Gloria

Ruiz-Hurtado, Gema

Ruiz-Moyano, Santiago

Ruml, Tomas

Runge, Kurt W.

Ruocco, Nadia

Rushworth, Stuart

Rusnati, Marco

Russo, Maria

Russo, Marina

Ruvo, Menotti

Ruysschaert, Jean Marie

Ruzov, Alexey

Ruzza, Chiara

Rybakowska, Iwona

Rybka, Krystyna

Rychkov, Grigori

Rzymowska, Jolanta

Saad, Mohamed
Saba, Julie

Sabatier, Jean-Marc

Sack, Ulrich

Saddala, Madhu Sudhana

Sádecká, Jana

SADOVSKAYA, Irina

Saele, Øystein

Sagheddu, Claudia

Saha, Sajib

Saha, Subhasish

Saha, Surya

Saheki, Takeyori

Sahni, Abha

Sahu, Pranav

Saiardi, Adolfo

Saikumar, Pothana

Saiman, Mohd Zuwairi

Saini, Ramesh Kumar

Sainlos, Matthieu

Saisho, Yoshifumi

Saito, Kosuke

Saito, Yoshinori

Saitoh, Kenji

Saitoh, Masao

Sakai, Hiromi

Sakai, Hiromichi

Sakamoto, Kensaku

Sala, Roberto

Sałaga, Maciej

Salata, Cristiano

Salazar, Ivan

Salehi, Hassan S.

Salehzadeh-Yazdi, Ali

Salerno, Loredana

Saller, Maximilian

Salman, Mootaz

Salvador, Armindo

Salvati, Erica

Salzano, Andrea

Salzer, Isabella

Salzet, Michel

Samaja, Michele

Samaniego, Rafael

Samarakoon, Rohan

Sambuceti, Gianmario

Šamec, Dunja

Samhan-Arias, Alejandro

Samiec, Marcin

Samson-Himmelstjerna, Georg Von

Samsonov, Sergey

Sanchez De Groot, Natalia 
Sánchez, Diego Hernán

Sanchez, Otto

Sanchez-Quesada, José Luis

Sanchiz, África

Sandbichler, Adolf

Sanders, Alison P.

Sanders, Yan

Sandström, Corinne

Sanejouand, Yves-Henri

Sanfeliu, Coral

Sanguinetti, Maurizio

Sanjeet, Mehariya

Sanjeewa, Kalu Kapuge Asanka

Santamaria, Rita

Santamaria, Salvatore

Santana, Andrés González

Santana-Codina, Naiara

Santiago-Vázque, Lory

Santin, Izortze

Santini, Antonello

Santonico, Elena

Santoni-Rugiu, Eric

Santos, Cleydson Breno R.

Santos, Hugo

Santos, Klebson

Santos, Maria M. M.

Santos, Raquel De Cássia Dos

Santoso, Netty

Santucci, Annalisa

Santulli, Gaetano

Sapozhnikov, Alexander M.

Sapudom, Jiranuwat

Sarasan, Viswambharan

Sarasua, Jose-Ramon

Sarkar, Amrita

Sarkar, Aurijit

Sarnataro, Daniela

Sartini, Davide

Sartorelli, Patricia

Sartorius, Kurt

Sasser, Jennifer M.

Sastre, Leandro

Sato, Kousuke

Sato, Shuzo

Satoh, Katsuya

Sattler, Wolfgang

Sauguet, Ludovic

Savchenko, Tatyana V.

Sazonova, Margarita A.

Scadding, Glenis Kathleen

Scampicchio, Matteo
Scanlon, Karen M.

Scarpa, Emanuele-Salvatore

Scarpa, Melania

Scarselli, Marco

Schaaper, Roel

Scheggi, Simona

Schelvis, Johannes

Schengrund, Cara-lynne

Schenone, Silvia

Scherf, Katharina A.

Schiefer, Ana Iris

Schiemann, Olav

Schild-Poulter, Caroline

Schindler, Charles W.

Schjoldager, Katrine T.-B.G.

Schmid, Diethart

schmidt, Florian

Schmidt, Kristina

Schmidtke, Gunter

Schmitz, Matthias

Schmitz, Ulf

Schneider, David

Schneider, Ian C.

Schneider, Robert

Schnell, Robert

Schoenberg, Daniel R.

Schoenhagen, Paul

Schohn, Hervé

Schols, Dominique

Scholtz, Vladimír

Schomacher, Lars

Schön, Jennifer

Schonewille, Martijn

Schrum, Adam

Schubert, Mario

Schubert, Stephanie

Schueler, Julia

Schultz, David J

Schulze-Tanzil, Gundula Gesine

Schwab, Rebecca

Schwaminger, Sebastian

Schwartz-Albiez, Reinhard

Scicchitano, Pietro

Scoffone, Viola Camilla

Scott, Ian

Scotti, Marcus

Scozzafava, Andrea

Scuruchi, Michele

Sebastiani, Federico

Sebestik, Jaroslav

Secci, Daniela 
Seco-Rovira, Vicente

Seeherunvong, Tossaporn

Seeliger, Jessica

Segura, Miguel F

Sek, Slawomir

Sekino, Yohei

Selent, Jana

Selivanova, Svetlana V.

Semina, Ekaterina V.

Senel, Mehmet

Sengupta, Bidisha

Seo, Daisuke

Sepuru, Krishna Mohan

Sequeda-Castañeda, Luis G.

Serefko, Anna

Sergeant, Kjell

Sergey, Netesov

Sergi, Consolato

Sergi, Domenico

Serino, Giovanna

Serrano, Juan Alfonso Ayala

Serrano, María

Serrano-Pertierra, Esther

Sethi, Gautam

Seto, Toshiyuki

Setzer, William N.

Sevastre, Bogdan

Sevilimedu Veeravalli, Sathyanarayanan

Sevvana, Madhumati

Seydel, Tilo

Sezgin, Erdinc

Sgambato, Alessandro

Sgherri, Cristina

Shackelford, Julia

Shackelford, Rodney E.

Shakya, Akhilesh

Shao, Fangwei

Shao, Peng

Sharma, Anket

Sharma, Bhesh Raj

Sharma, Isha

Sharma, Piyush Sindhu

Shaul, Yoav D.

Shaw, Pang-Chui

Shaw, Robin M.

Shcherbakova, Daria

Shcherban, Andrey B.

Shchyogolev, Sergei

She, Zhi-Gang

Shee, Kevin

Shellaiah, Muthaiah
Shen, Haihong

Shen, Long

Shen, Qiang

Shen, Weining

Shen, Xiulong

Shen, Yi

Shen, Yulong

Sheng, Yi

Shi, Junchao

Shi, Lei

Shi, Yanshu

Shi, Zengqian

Shi, Zhi

Shiao, Young-Ji

Shibata, Hideki

Shidoji, Yoshihiro

Shieh, Tzong-Ming

Shifman, Julia

Shigeta, Yasuteru

Shih, Chun-Kuang

Shih, Yin-Hwa

Shikama, Yosuke

Shikov, Alexander

Shim, Hyunbo

Shimada, Yasuhito

Shimizu, Katsuhiko

Shimizu, Takahiko

Shinohara, Minoru

Shin-Yi, Marzano

Shiomi, Yasushi

Shiraishi, Fumito

Shirakawa, Tetsuo

Shirato, Ken

Shirole, Nitin

Shiuchi, Tetsuya

Shoshan-Barmatz, Varda

Shriver, Leah

Shugo, Suzuki

Shukla, Kirtikar

Shukla, Sanjay K.

Shvets, Elena

Siegel, Amanda

Sieira, Rodrigo

Sieniawska, Elwira

Sikazwe, Donald

Šikurová, Libuša

Silachev, Denis

Sileikyte, Justina

Silman, Israel

Silva, Alexandre R

Silva, Andre 
Silva, Susana N.

Silva-Cunha, Armando

Sim, Jaehoon

Simanshu, Dhirendra K

Simirgiotis, Mario

Simões, Nelson

Simon, Istvan

Simon, Jorge

Simon, Liz

Simon, Michel

Simone, Anfossi

Simone, Angela De

Šimoník, Ondřej

Simonsson, Stina

Sinanoglou, Vassilia

Šindlerová, Lenka

Singh, Anand

Singh, Anil

Singh, Brijesh Kumar

Singh, Somnath

Singla, Bhupesh

Sinharoy, Pritam

Siniscalco, Dario

Šinko, Goran

Sintim, Herman

Siódmiak, Jacek

Sipos, Katalin

Siristatidis, Charalampos

Sissler, Marie

Sisto, Margherita

Sitdikova, Guzel

Sivaraj, Kishor Kumar

Skała, Ewa

Skandalis, Spyros

Skorko-Glonek, Joanna

Skorska, Anna

Skowyra, Dorota

Skrypnik, Katarzyna

Skrzypek, Klaudia

Skrzypski, Marek

Skuja, Sandra

Skupin-Mrugalska, Paulina

Skurikhin, Evgenii Germanovich

Skvortsov, Dmitry

Slatter, M. A.

Sleigh, Alison

Slim, Smaoui

Śliwińska-Wilczewska, Sylwia

Słomińska-Wojewódzka, Monika

Slominski, Andrzej

Słomka, Artur
Słomko, Joanna

Smalley, Joshua L.

Smeriglio, Antonella

Śmieszek, Agnieszka

Smith, David

Smith, Eric

Smith, Jeffrey

Smith, Jennifer

Smith, Jeremy C.

Smith, Micholas Dean

Smith, Robert E.

Smits, Kim M.

Smolková, Katarina

Snider, Ashley J

Snider, Mark J.

Snowden, Timothy

Soares, Raquel

Sobeh, Mansour

Sobieszczuk-Nowicka, Ewa

Sobol, Robert

Sobrado, Pablo

Sodi, Anna Mensuali

Sodt, Alexander J.

Soini, Sanna

Soleimanpour, Arash S.

Soler, Miguel Ángel

Solingapuram Sai, Kiran Kumar

Soltész, Alexandra

Sompol, Pradoldej

Soncin, Francesca

Song, Kenan

Song, Myoung-Chong

Song, Yuyu

Soond, Surinder M.

Soós, Vilmos

Sophianopoulou, Vicky

Soran, Maria-Loredana

Sorensen, John

Sorescu, Ana Alexandra

Sorgen, Paul L.

Sorrell, J. Michael

Sorrenti, Valeria

Sorsa, Timo

Soslau, Gerald

Soto, Robert

Soufan, Othman

Soulimane, Tewfik

Soundararajan, Prabhakaran

Sousa, Maria João

Sousa, Sérgio F.

South, Paul F. 
Sovadinova, Iva

Soveral, Graça

Sowa, Yoshiyuki

Soyfoo, Muhammad

Spagnol, Gaëlle

Speijer, Dave

Spella, Magda

Spence, Rowena

Spencer, Gaynor E.

Spies, Maria

Spittau, Björn

Splichal, Igor

Spratt, Donald E.

Spray, David

Spur, Bernd W.

Średnicka-Tober, Dominika

Sridhar, Jayalakshmi

Srivastava, Pranay

Srivastava, Sameer

Srivastava, Saurabh

Staal, Jens

Stadlbauer, Petr

Staiger, Dorothee

Stana, Anca - Daniela

Stanek, Agata

Stanford, Stephanie

Stange, Katja

Stanisz, Beata

Stankovic, Milan

Stanley, Jone A.

Stanley, Robin

Stano, Pasquale

Starek, Małgorzata

Statti, Giacarlo

Stear, Michael

Stefanou, Stefanos

Stefanowicz, Joanna

Steger, Gerhard

Steinbeck, Christoph

Steiner, Jennifer

Steiner, Joseph P.

Stellato, Francesco

Stenbeck, Gudrun

Stepanenko, Olesya V.

Stepanova, Anna A.

Stephens, Gary

Stephens, Jacqueline M.

Sterckx, Yann

Steri, Maristella

Sterling, James D.

Sterling, Julie
Sterner, Olov

Steuer, Christian

Stewart-Ornstein, Jacob

Sticht, Heinrich

Stilhano, Roberta S.

Stimamiglio, Marco Augusto

Stines-Chaumeil, Claire

Stinghen, Andréa Emilia Marques

Stoean, Catalin

Stojakowska, Anna

Stojanov, Milos

Stojko, Jerzy

Stojko, Rafał

Stone, Kari L.

Stone, Trevor W.

Stone, William L.

Stoppelkamp, Sandra

Stork, Christian J.

Storr, Tim

Stoyanova, Albena

Stradner, Martin Helmut

Straight, Paul

Strappazzon, Flavie

Sträter, Norbert

Strati, Irini F.

Straus, Suzana K.

Stravopodis, Dimitrios J.

Strehmel, Veronika

Strelnikov, Vladimir V.

Struzik, Justyna

Strzelecki, Dominik

Stuart, David T.

Su, Jing

$\mathrm{Su}$, Jiyong

$\mathrm{Su}, \mathrm{Mei}-\mathrm{Tsz}$

Su, Zhaoqian

$\mathrm{Su}$, Zheng-Yuan

Suarato, Giulia

Suciu, Bogdan Andrei

Sugawara, Akihiro

Sugaya, Makoto

Sugii, Hideki

Sugimoto, Keiichiro

Sugimoto, Masahiro

Suh, Hyung Joo

Suleria, Hafiz Ansar Rasul

Sullivan, John Stephen

Sumara, Grzegorz

Sumbayev, Vadim

Sun, Chaomin

Sun, Guoxing 
Sun, Lichun

Sun, Wei

Sun, Zhixiong

Sun, Zhonghua

Sunayama, Hirobumi

Sundaresan, Alamelu

Sundberg, Eric J.

Suñer, Damian Heine

Suomela, Jukka-Pekka

Superti, Fabiana

Sur, Subhayan

Surguchov, Andrei

Sushchik, Nadezhda N.

Suwinska, Kinga

Suzui, Masumi

Suzuki, Ayako

Suzuki, Keiko

Suzuki, Shinya

Suzuki, Toru

Svahn, Ola

Svendsen, John S.

Svetashev, Vasily

Swairjo, Manal A.

Swarbrick, Crystall

Sweeney, Elizabeth

Świderek, Katarzyna

Swietnicki, Wieslaw

Swiezewska, Ewa

Sykłowska-Baranek, Katarzyna

Sýkora, Jan

Synnott, Naoise

Syrovets, Tatiana

Sysolyatina, Elena

Szakiel, Anna

Szaleniec, Maciej

Szatmari, Erzsebet Maria

Szatmári, Tünde

Szavits-Nossan, Juraj

Szczepanek, Joanna

Szczepanik, Antoni M

Szczepankiewicz, Aleksandra

Szeberény, József

Szegletes, Zsolt

Szekalska, Marta

Szeliga, Jacek

Szentmary, Nora

Szolnoky, Gyozo

Szperlik, Jakub

Szuts, David

Szweda, Piotr

Szweda, Roza
Szymańki, Paweł

Szymczyk, Agnieszka

Tachezy, Jan

Tae, Han-Shen

Tafelska-Kaczmarek, Agnieszka

Tafuri, Simona

Tagliavia, Marcello

Tagliazucchi, Davide

Taguchi, Yoshihiro

Taha, Muhammad

Tahara, Hidetoshi

Tahirov, Tahir H.

Tailhades, Julien

Taillandier, Daniel

Tajiri, Kazuto

Takahashi, Reisuke H.

Takahashi, Toshio

Takaki, Manabu

Takano, Katsura

Takasawa, Shin

Takeda, Sen

Takeuchi, Hideyuki

Taki, Masumi

Talavera, Miguel

Talbot, Jeffery N.

Talhinhas, Pedro

Talukdar, Sarmistha

Taly, Antoine

Tam, James P.

Tamasi, Gabriella

Tambaro, Simone

Tambe, Mitali

Tamkovich, Svetlana

Tan, Du-Xian

Tan, Yaw Sing

Tanabe, Shihori

Tanaka, Junichi

Tanaka, Naoki

Tanaka, Nobutada

Tanaka, Takuji

Tanase, Corneliu

Tanase, Cristiana

Tanc, Muhammet

Tang, Aihua

Tang, Bor Luen

Tang, Jing

Tang, Renjie

Tang, Soon Yew

Tang, Xiangru

Tani, Akio

Tanyi, Janos L. 
Tao, Peng

Tao, Yongsheng

Tarafder, Solaiman

Tarasov, Andrei

Tarn, Woan-Yuh

Tashima, Kimihito

Tashima, Toshihiko

Tata, Ada Maria

Tate, Shin-Ichi

Tatenhorst, Lars

Tatsuta, Takashi

Tatulian, Suren A.

Tatullo, Marco

Taupitz, Matthias

Tavanti, Francesco

Tavares, Joana

Tavolari, Simona

Tawara, Ken

Taylor, Eric B.

Tchetina, Elena V.

Tchoghandjian-Auphan, Aurélie

Tchórzewski, Marek

Tedeschi, Valentina

Teisseyre, Andrzej

Teixeira, Ana Luísa

Teixeira, Fábio G.

Teixeira, Miguel

Teklić, Tihana

Téllez-Téllez, Maura

Tellier, Michael

Temeyer, Kevin B.

Temussi, Piero A.

Teng, Yong

Terao, Mineko

Terashima, Hiroyuki

Terme, Nolwenn

Terrinoni, Alessandro

Terryn, Christine

Terzopoulou, Zoe

Teske, Andreas

Tesmer, John

Than, Nandor Gabor

Thanabalu, Thirumaran

Thet, Naing

Thevenod, Frank

Thiel, Kristina

Thomas, Wayne

Thomes, Paul

Thompson, Damien

Thoms, Sven

Thor, Stefan
Thorpe, Stephen

Thounaojam, Menaka Chanu

Thummel, Ryan

Thurnher, Martin

Tian, Sheng

Tian, Ya-Chung

Tian, Yuan

Ticconi, Carlo

Tiera, Marcio José

Tikhonova, Irina

Timoshenko, Alexander V.

Timson, David J.

Tinti, Anna

Tisné, Carine

Tiso, Natascia

Tixeira, Rochelle

Tkac, Jan

Tocco, Graziella

Toda, Tomohisa

Todaka, Daisuke

Todea, Anamaria

Togashi, Yuichi

Toiu, Anca

Toma-Fukai, Sachiko

Tomar, Dhanendra

Tomaszewska, Emilia

Tomatsu, Shunji

Tomczykowa, Monika

Tomi, Félix

Tomikawa, Chie

Tomko, Robert J.

Toms, Derek

Tonello, Fiorella

Tong, Hongning

Tong, Junchao

Tong, Xinming

Tong, Yaojun

Topin, Jeremie

Toppo, Stefano

Tordai, Attila

Torres Vaamonde, José Enrique

Torres, Adrian Gabriel

Torres-Lagares, Daniel

Torres-Ramírez, Eduardo

Toto, Angelo

Totoson, Perle

Toubanaki, Dimitra

Tõugu, Vello

Toy, Randall

Trackman, Philip C.

Trader, Darci 
Traiffort, Élisabeth

Trakselis, Michael A.

Tran, Anh Nhat

Tratnik, Janja Snoj

Traw, Brian

Trček, Janja

Treebak, Jonas

Treinin, Millet

Tresguerres, Jesus

Tribulova, Narcis

Trincone, Antonio

Tripathi, Jitendra Kumar

Tríska, Jan

Trivedi, Darshan

Tronchere, Helene

Trouvelot, Sophie

Trubiani, Oriana

Trudel, Dominique

Truman, James W.

Trusheva, Boryana

Truzzi, Cristina

Truzzi, Daniela R.

Tsafantakis, Nikolaos

Tsai, Ang-Chen

Tsai, Kun-Lin

Tsai, Pei-Shiue

Tsai, Pei-Yin

Tsai, Shu-Yao

Tsapogas, Panagiotis

Tsarouhas, Konstantinos

Tse Seng, Chuah

Tseng, Chih-Hua

Tsermpini, Evangelia Eirini

Tsoi, Ho

Tsotinis, Andrew

Tsouh Fokou, Patrick Valere

Tsuchiya, Yuichi

Tsuda, Hiroyuki

Tsuda, Sakae

Tsugawa, Hitoshi

Tsuge, Tomohiko

Tsujita, Maki

Tsukahara, Toshifumi

Tsumuraya, Takeshi

Tsuruta, Daisuke

Tuccinardi, Tiziano

Tucker, Julie

Tucker, Nathan

Tudela, Jose

Tufarelli, Vincenzo

Tuleja, Monika
Tung, Tao-Hsin

Tupally, Karnaker R.

Tupler, Rossella

Turano, Paola

Turk, John

Turner, Mark

Tuukkanen, Juha

Tylova, Edita

Tyson-Capper, Alison

Tytgat, Jan

Tzen, Jason T.

Tzortzakis, Nikos

Tzovenis, Ioannis

Uchida, Léo

Uchiyama, Taketo

Uhrig, R. Glen

Uldrich, Adam

Uldrijan, Stjepan

Ulicna, Livia

Ullah, Irfan

Ullah, Mujib

Umar, Shahid

Ungaro, Federica

Unno, Hideaki

Unoki, Takamitsu

Upham, Brad L.

Urban, Milan

Urban, Philippe

Urbanc, Brigita

Urbanelli, Lorena

Urbaniak, Alicja

Urbanowicz, Marcin

Urbanska, Edyta Maria

Urra, Félix A.

Usuki, Yoshinosuke

Uversky, Vladimir

Uygun, Sahra

Uzbekova, Svetlana

Vaamonde, Carlos

Vaidotas, Stankevičius

Vaidyanathan, Venkatesh

Vakonakis, John

Valdés López, Oswaldo

Valenti, Maria

Valentim, Ana

Valentine, Rudy J.

Valenzuela, Juan Luis

Valis, Martin

Vallera, Daniel

Valsami, Georgia

Van Aerschot, Arthur 
Van Delft, Floris L.

Van Den Ende, Wim

Van Der Nest, Magriet A

Van Der Vegt, Bert

Van Doorn, Remco

Van Haute, Lindsey

Van Helden, Dirk

Van Hellemond, Jaap J.

Van Houten, Judith

Van Lith, Sanne

Van Raaij, Mark

Van Rossum, Huub H.

Van Waardenburg, Robert

Vance, David

Vanderlei, Maria De Fátima

Vandooren, Jennifer

Vangala, Janakiram R.

Vanharanta, Sakari

Vanin, Anatoly F.

Vaquero, Javier

Varadarajan, Shankar

Váradi, Csaba

Váradi, Györgyi

Varga, Zoltan M.

Vargas Jentzsch, Paul

Vargas-Murga, Liliana

Varikuti, Sanjay

Vaseva, Irina I.

Vasic, Vesna

Vassallo, Neville

Vassão, Daniel Giddings

Vasylieva, Natalia

Vatrella, Alessandro

Vaz, Daniela C.

Vazquez, Alexei

Vazquez, Elba S.

Veeramani, Suresh

Vega, Juan Francisco

Végh, Attila Gergely

Veit, Michael

Veldkamp, Christopher

Velgosova, Oksana

Vellecco, Valentina

Vellido, Alfredo

Venditto, Vincent

Venkatesan, Jagadeesh K

Venkateswaran, Suresh

Vera Ávila, Héctor Raymundo

Verde, Federico

Verdeguer, Francisco

Vergara, Daniele
Verma, Rajni

Verma, Ranjit

Verma, Rati

Vermaas, Joshua V.

Verna, Roberto

Verreault, Maïté

Versacci, Paolo

Verspoor, Karin

Verzola, Daniela

Veschi, Veronica

Vetere, Amedeo

Vetrano, Stefania

Vibhute, Sandip M.

Vicario-Abejón, Carlos

Vicens, Quentin Jerome

Vicente, Laura

Vicente-Manzanares, Miguel

Victor, Bruno

Victor, Victor

Victoria - Clos, M.

Viegas, Sandra C.

Vieira, Alexandre

Viennet, Thibault

Vigetti, Davide

Víglaský, Viktor

Vignini, Arianna

Vignoli, Alessia

Vigodner, Margarita

Viguera, Ana Rosa

Vijgenboom, Erik

Viktorova, Jitka

Vilela, Alice

Vilela, Janice Miranda Vasconcellos

Villalobo, Antonio

Villar-Piqué, Anna

Villegas-Sepulveda, Nicolas

Vimalachandran, D.

Vimberg, Vladimir

Viña, Dolores

Vincent, Delphine

Vinet, Raúl

Vingolo, Enzo Maria

Vinha, Ana

Vinjamur, Divya

Viola, Ivana

Virag, Jitka A.I.

Virgili, Fabio

Vishe, Mahesh

Vitetta, Luis

Vito, Pasquale

Vitoratos, Evangelos 
Vitrac, Heidi

Vives, Joaquim

Vives-Peris, Vicente

Vizovišek, Matej

Vlachou, Marilena

Vlková, Eva

Voběrková, Stanislava

Vogel, Alexander

Vögele, Martin

Vogt, Martin

Volkov, Vadim

Vondracek, Jan

Vorontsov, Dmitry

Voss, Matthias

Vozella, Valentina

Vulturar, Romana

Wada, Jun

Wadhwa, Navish

Wagner, Kay Dietrich

Wagner, Renaud

Wahli, Walter

Wahlsten, Matti

Wakao, Masahiro

Wako, Hiroshi

Wałajtys-Rode, Elżbieta

Waldum, Helge

Walker, Anthony L.

Walker, Douglas G.

Walker, Mary K.

Walker, Robert J.

Wall Medrano, Abraham

Wallace, Heather M.

Walsh, Laurence J

Walter, Jeske

Wan, Lei

Wan, Shibiao

Wan, Zhongxiao

Wanders, Ronald J.A.

Wang, Be-Jen

Wang, Bin

Wang, Chao

Wang, Cheng

Wang, Chin-Kun

Wang, Chun

Wang, Di-Yan

Wang, Guliang

Wang, Huaishan

Wang, Huawei

Wang, Jee-Ching

Wang, Jianjun

Wang, Jimin
Wang, Jin'An

Wang, Nan

Wang, Ning

Wang, Ruiyong

Wang, Sai

Wang, Tianfang

Wang, Wenqi

Wang, Xiumin

Wang, $\mathrm{Xu}$

Wang, Yan

Wang, Yeng-Tseng

Wang, Yifei

Wang, Yongqiang

Wang, Zhen

Wang, Zhiqiang

Ward, Robert

Ward, Robert M.

Wärmländer, Sebastian K.t.s.

Warzych-Plejer, Ewelina

Wasan, Ellen

Washino, Satoshi

Watanabe, Yoichiro

Wautier, Jean-Luc

Waxman, Joshua

WAYE, Mary M.Y.

Webb, R. Clinton

Webba Da Silva, Mateus

Weber, Daniel N.

Webster, Keith A

Wei, Kongchang

Wei, Shuo

Weidenhofer, Judith

Weinhold, Alexander

Weinzierl, Robert

Weiser, Brian P.

Weitzman, Matthew

Welch, Ailsa

Weldon, John

Weldy, Chad S.

Welling, Mick

Wells, Laura

Weltin, Andreas

Wemmer, David

Wen, Yu-Ching

Wen, Zhiwei

Wenda-Piesik, Anna

Weng, Mao-Lun

Westh, Peter

Weston, Leslie A.

Weydt, Patrick

Whatmore, Jacqueline 
Whelan, Derek

White, Carl

White, Robert

Whitehead, Shawn N.

Whitfield, Phil.

Wicky Collaud, Chantal

Wie, Myung-Bok

Wieckowska, Anna

Wieczorek, Edyta

Wiedemann, Nils

Wiener, Reuven

Wiese, Sebastian

Wiesman, Zeev

Wiesner-Reinhold, Melanie

Więsyk, Aneta

Wightman, Emma L.

Wiglusz, Katarzyna

Wijnveld, Michiel

Wildemann, Britt

Wilkinson, Kevin A

Wilkinson, Miles F.

Wilkowska, Agnieszka

Willemse, Eline

Williams, Daniel

Williams, Frederick

Williams, Jesse

Williams, Marshall

Williams, Michelle

Williamson, Ethel Diane

Wilson, Jeremy Somers

Wilson, Peter

Wiltschi, Birgit

Wimmer, Monika

Wimmer, Zdenek

Windshügel, Björn

Winkle, Melanie

Wińska, Katarzyna

Wirz, Richard E.

Witte, Angela

Wittig, Ilka

Wlaź, Piotr

Wnorowski, Artur

Wnuk, Maciej

Wnuk, Stanislaw

Woerman, Amanda

Wójciak, Magdalena

Wojtunik-Kulesza, Karolina Anna

Wolf, Phillipp

Wołkow, Paweł

Wong, Chi-Ming

Wong, Ching-On
Wong, Maurice

Wong, Yung-Sing

Wong, Yvette

Wood, David W.

Woolf, Eric J.

Worch, Remigiusz

Worman, Howard

Worsnop, Christopher J

Worthmann, Anna

Woscholski, Rudiger

Wöstemeyer, Johannes

Wozniak-Knopp, Gordana

Wree, Andreas

Wright, Gerard D.

Wróbel, Maria

$\mathrm{Wu}$, (Jason) Boyang

$\mathrm{Wu}, \mathrm{Cen}$

$\mathrm{Wu}$, Chung-Hsin

Wu, Chung-Yi

$\mathrm{Wu}$, Erxi

$\mathrm{Wu}$, Jennifer

$\mathrm{Wu}$, Jin-Ming

$\mathrm{Wu}$, Li-chen

$\mathrm{Wu}$, Long-fei

$\mathrm{Wu}$, Meng

$\mathrm{Wu}$, Ming-Jiuan

$\mathrm{Wu}$, Min-Huan

$\mathrm{Wu}$, Ping-Hsun

$\mathrm{Wu}$, Yingnian

$\mathrm{Wu}$, Youjun

$\mathrm{Wu}, \mathrm{Yu}$-Jen

$\mathrm{Wu}$, Zhihao

Wurm, Jan-Philip

Wyszkowski, Mirosław

Xavier, Nuno M.

$X i$, Shuhua

$\mathrm{X}_{\mathrm{i}}$, Xinping

Xia, Jun

Xiao, Xiangwei

Xie, Bingxian

Xie, Min

Xie, Mouzhe

Xie, Ting

Xie, Wei-dong

Xie, Wenjun

XIRODIMAS, Dmitris

$\mathrm{Xu}, \mathrm{Kui}$

Xu, Peng

$\mathrm{Xu}$, Shengli

$\mathrm{Xu}$, Wenyan

$\mathrm{Xu}, \mathrm{X}$ in 


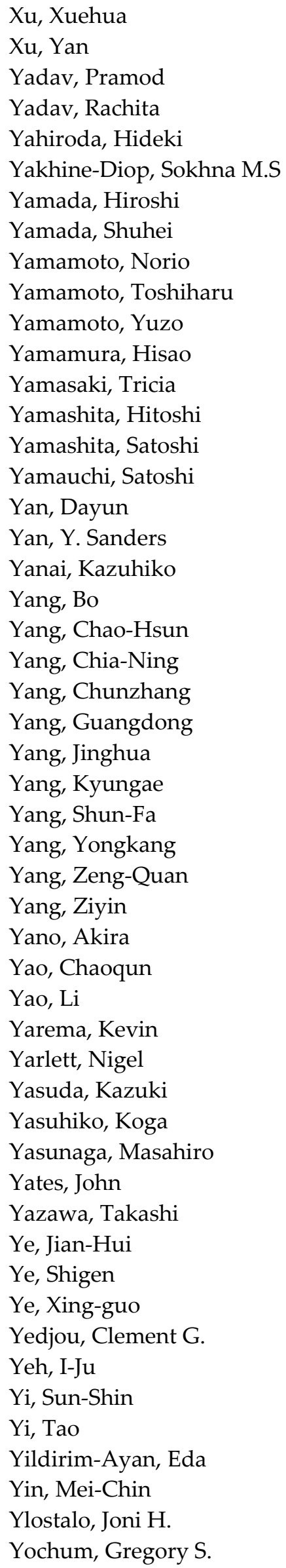

Yohda, Masafumi

Yokoi, Fumiaki

Yonekura, Shinichi

Yoon, Joon-Kee

Yoon, Yisang

Yoshikawa, Kenichi

Yoshimatsu, Takeshi

Yoshimura, Masami

You, Youngjae

Younger, Scott

$\mathrm{Yu}$, Cheng-Ju

Yu, Danxia

Yu, Deng-Guang

Yu, Han-Gang

Yu, Jian

$\mathrm{Yu}$, Lu-Gang

Yu, Tsyr-Yan

$\mathrm{Yu}$, Victor

Yu, Yu-Hsiang

Yuan, Gailing

Yuan, Qingbin

Yuan, Xuegang

Yuan, Zhihong

Yuann, Jeu-Ming P.

Yucesan, Gundog

Yudoh, Kazuo

Yuguchi, Yoshiaki

Yukl, Erik T.

Yumoto, Hiromichi

Yurchenko, Ekaterina

Yurina, Nadezhda P.

Yutzey, Katherine

Zacconi, Flavia

Zagotto, Giuseppe

Zaharia, Valentin

Zahoranová, Anna

Zaichenko, Olexandr S.

Zaid, Hilal

Zakiyanov, Oskar

Zakrocka, Izabela

Zalewska, Anna

Zalubovskis, Ravis

Zam, Wissam

Zambito, Ylenia

Zambrano, Angara

Zamfir, Medana

Zamparini, Fausto

Zanotti, Giuseppe

Zapico, Sara C.

Zappavigna, Silvia

Zappulla, David 
Zarschler, Kristof

Żarski, Daniel

Zarza, Xavier

Zawacka-Pankau, Joanna

Zeigler, Daniel

Zeković, Zoran

Żelaszczyk, Dorota

Zelkó, Romána

Zenarruzabeitia, Olatz

Zhang, Ershuai

Zhang, Fan

Zhang, Fuming

Zhang, Ji

Zhang, Jian

Zhang, Jianying

Zhang, Pengfei

Zhang, Quan

Zhang, Wencai

Zhang, Wenxuan

Zhang, Xiaodong

Zhang, Xiaonan

Zhang, Yixuan

Zhang, Yonghong

Zhang, Zhao

Zhang, Zhibing

Zhang, Zhiguo

Zhao, Huixian

Zhao, Ling

Zhao, Ning

Zhao, Ningning

Zhao, Qian

Zhao, Yang

Zhao, Yuguang

Zhao, Yutong

Zheng, Wenwei

Zheng, Yun-Wen

Zheng, Zhong

Zhou, Bangjun

Zhou, Chi

Zhou, Hao

Zhou, Heshan Sam

Zhou, Hua

Zhou, Jinrong
Zhou, Liang

Zhou, Min

Zhou, Phoebe

Zhou, Qimin

Zhou, Qingyu

Zhou, Tianhao

Zhou, Yubin

Zhou, Zhidong

Zhu, Lin

Zhu, Pengxiang

Zhu, Qiuyu Martin

Zhu, Quinn

Zhu, Shiwei

Zhu, Shujia

Zhu, Wufu

Zhukov, Igor

Zhukova, Natalia

Zhuo, Chunliu

Zidar, Nina

Zidek, Jan

Ziemann, Mark

Zille, Andrea

Zimmermann, Sonja

Zinta, Gaurav

Zizza, Pasquale

Zloh, Mire

Złotkowska, Dagmara

Zmijewski, Michal

Zographos, S. E.

Zoidl, Georg

Zolfaghari Emameh, Reza

Zondler, Lisa

Zórad, Stefan

Zou, Xueqing

Zovko Končić, Marijana

Zubair, Abba C.

Zunk, Matthew

Zupan, Janja

Zuppinger, Christian

Zuverza-Mena, Nubia

Zuza, Ester

Zylinska, Ludmila 